ATLAS Internal Note INDET-NO-???

September 18, 1998

\title{
X-Ray Tomography for the ATLAS Semi-Conductor Tracker
}

\author{
J.Bibby, J.Grosse-Knetter, R.Nickerson, L.Vertogradov* \\ Particle \&o Nuclear Physics \\ Oxford University \\ Oxford OX1 3RH, UK
}

\begin{abstract}
Results are presented of methodical investigations of an X-ray tomography system for positioning silicon strip modules. The achieved efficiency and a measured accuracy of better than $5 \mu \mathrm{m}$ is sufficient for using this method in a high precision survey of the ATLAS inner tracker.
\end{abstract}

*also at JINR, Dubna, Russia. 


\section{Introduction}

In the ATLAS inner tracker, large area multilayer structures must be aligned with an accuracy better than the intrinsic resolution of their detecting elements. The requirements range from 7 to $20 \mu \mathrm{m}$. Laser and opto-electronic systems are used for measuring and monitoring changes in the relative positions between different elements. The X-ray scanning technique is well suited to high precision survey of the position of sensitive elements. This survey procedure (coordinate calibration) is used as a final step in the detector production chain. The alignment systems use the survey as a zero point for detector alignment. The alignment and survey strategy for the inner detector is documented in [1].

For alignment and coordinate calibration of wire chambers scanning with a narrow X-ray beam has been used [2] and is developed here for use with silicon tracking detectors. The technique is based on two commercially available components:

- Fine focus spot X-ray tubes ("Europe Standard Diffractive Tubes") with $\delta_{\text {focus }}=100$ to $400 \mu \mathrm{m} ; U=20$ to $60 \mathrm{kV} ; P=1$ to $2 \mathrm{~kW}$;

- High accuracy and resolution linear and rotary stages: $\sigma_{x}=1$ to $10 \mu \mathrm{m}$; $\sigma_{\phi} \leq 1^{\prime \prime}$ in the range from $0^{\circ}$ to $360^{\circ}$.

The low wavelength of photons used $(\lambda=0.01$ to $0.05 \mathrm{~nm})$ allows the formation of narrow beams $\left(\delta_{\text {beam }}=20\right.$ to $\left.100 \mu \mathrm{m}\right)$ over a distance of a few metres without any diffraction problems. A sufficiently small divergence $\left(\partial \delta_{\text {beam }} / \partial D \approx\right.$ $0.2 \mathrm{mrad} \approx 40^{\prime \prime}$ ) can be attained by collimating slits.

The silicon modules in the Semi-Conductor Tracker (SCT) can be used for an active mode of the X-ray survey. This means that the silicon module itself is used for counting X-ray quanta which then allows an accurate reconstruction of the module position. This is based on the direction of the X-ray which is precisely defined by the linear or rotary stage. To develop this method an Xray scanning facility (XTomo1D) was constructed. This is the first phase of a three step programme leading to a full three-dimensional survey machine for the ATLAS SCT. The next two sections give a technical description of XTomo1D and present measurment results.

\section{X-ray Scanning Facility}

A general view of the X-ray facility is shown in the photograph in figure 1 . The scanning device itself is presented in the second photograph in figure 2 and its drawing in figure 3. Data collection and scanning is controlled by a local computer. 
The coordinate system of the scanning device referred to later is indicated in figure 3. The $Y$ axis is parallel to the $\mathrm{X}$-ray beam and the $Z$ axis perpendicular to it such that it is parallel to the final collimator slit. The $X$ axis is perpendicular to both such that a right-handed system is formed.

\subsection{Hardware}

The XTomo1D apparatus has the following hardware components:

- X-ray high tension power supply: type VIP2-50-60M ${ }^{1}$, two outputs, max $50 \mathrm{kV}, 60 \mathrm{~mA}, 1 \%$ stability; dimensions $120 \times 90 \times 65 \mathrm{~cm}^{3}$; weight $\sim 400 \mathrm{~kg}$.

- X-ray tube: BSV-21ㄹ, W or Mo anode material, max $60 \mathrm{kV}, 20 \mathrm{~mA}$; focus spot dimensions $l \times \delta=8 \times 0.4 \mathrm{~mm}^{2}$; four output Be windows in the range of $0^{\circ}$ to $12^{\circ}$ relative to the anode working surface; water cooling with a flow of $3 \ell / \mathrm{min}$.

- $X-Z$ scanning table composed from two identical linear stages: Series 1000 Linear Position Stages ${ }^{3}$, travelling distance $50 \mathrm{~mm}$, resolution $1 \mu \mathrm{m}$, accuracy $4 \mu \mathrm{m}$ on $25 \mathrm{~mm}$ travel distance; control card PC23.

- Double sided Si strip module: $300 \mu \mathrm{m}$ thickness, $\Delta x=50 \mu \mathrm{m}$ readout pitch on one side, $\Delta z=150 \mu \mathrm{m}$ on the other from DELPHI Microvertex Detector [5]. It was installed horizontally inside a lightproof box with two $25 \mu \mathrm{m}$ Al-foil windows for the X-ray beam on either side. The box was fixed on top of the scanning table. Its position around the vertical axis could be tuned by a micrometer head in a range of $\pm 1.8^{\circ}$ with a resolution of $0.004^{\circ}$.

- Scintillation counter: plastic, dimensions $500 \times 78 \times 20 \mathrm{~mm}^{3}$, phototube RCA 8575.

\subsection{X-ray Beam Collimation}

The collimating scheme is shown in figure 4 . The source spot measures $8 \times 0.4 \mathrm{~mm}^{2}$ on the anode surface. The spot is viewed at an oblique angle, reducing the effective source size in one dimension. Only one window of the X-ray tube is used.

The final collimating slit has a tantalum inner surface and is placed at a distance $B \approx 200 \mathrm{~mm}$ along the $Y$ axis from the focus spot. It defines the width of the beam in $X$ direction. The width, $s$, of the slit can be tuned manually with a micrometer head from 0 to $1000 \mu \mathrm{m}$ with $10 \mu \mathrm{m}$ resolution.

\footnotetext{
${ }^{1}$ BUREVESTNIK firm, St Petersburg, Russia, 1982.

${ }^{2}$ SVETLANA firm, St Petersburg, Russia, 1997 [3].

${ }^{3}$ Naples Coombe Ltd., Chaddleworth, UK, 1996 [4].
} 
Lead masks restrict the output acceptance of the tube and define the length of the X-ray beam cross section along the $Z$ axis. The beam divergence in $X$ direction is equal to $\Delta \theta=\left(s+\delta_{X}\right) / B$, where $\delta_{X}$ is an imaginary width of the focus spot projected on the $X-Z$ plane. The small value of $3^{\circ}$ of the angle between anode surface and beam direction provides a $\delta_{X}$ being approximately 20 times smaller than the real width $\delta$ (see figure 4 ):

$$
\delta_{X}=\delta \cdot \tan 3^{\circ} \approx 400 \mu \mathrm{m} / 20=20 \mu \mathrm{m} .
$$

Thus, for $s=20 \mu \mathrm{m}$ the divergence of the beam is estimated to:

$$
\Delta \theta \approx(20 \mu \mathrm{m}+20 \mu \mathrm{m}) / 200 \mathrm{~mm} \approx 200 \mu \mathrm{rad} .
$$

Simple geometrical calculations of the beam intensity profile are shown in figure 5 . The half-width $c$ of the peak detected by the Si module is given by:

$$
c=s \cdot(B+D) / B,
$$

where $D=50 \mathrm{~mm}$ is the distance from the collimating slit to the Si module. In practice, $c$ values ranging between 25 and $250 \mu \mathrm{m}$ were used.

\section{X-ray Rates}

\subsection{Raw X-ray Spectrum}

The primary bremsstrahlung X-ray energy spectrum is approximated by the formula [6]:

$$
I\left(E_{\gamma}\right)= \begin{cases}\frac{2 I_{0}}{V^{2}}\left(V-E_{\gamma}\right), & \text { if } E_{\gamma}<V \\ 0 & \text { otherwise }\end{cases}
$$

where $E_{\gamma}$ is the X-ray quanta energy in eV, $I_{0}$ is the total intensity, and $V$ the high voltage at the anode of the X-ray tube. The attenuation of X-rays in matter is given by the X-ray attenuation coefficient $\mu\left(E_{\gamma}\right)$ of the material [7] (shown in figure 6 for silicon):

$$
I_{t r}\left(E_{\gamma}\right)=I\left(E_{\gamma}\right) \cdot \mathrm{e}^{-t \mu\left(E_{\gamma}\right)},
$$

where $I_{t r}$ is the intensity after traversing through matter of thickness $t$. The intensity deposited in matter is then given by $I_{d}=I-I_{t r}$.

\subsection{Scintillation Counter}

The rate of the discriminated pulses with and without X-ray beam for different voltages $U$ driving the phototube is shown in figure 7 . The curve without beam, ie reflecting the noise from the tube and cosmic background, is well distinguished from the signal. The signal to noise ratio is best at $U=2700 \mathrm{~V}$, which was used as the working voltage. 


\subsection{Si Strip Detector}

Unlike charged particles, photons are absorbed when detected in the Si module. As a consequence no externally driven triggering is possible. Instead, random triggering was used. After a start signal from the computer a data taking procedure is initialised. The detecting and readout cycle was repeated several times with a rate of approximately $20 \mathrm{~Hz}$. Electric charge collected during the detecting phase is treated as a "signal".

Without X-ray beam, the average of the signals of 100 measurements defines a pedestal while the RMS can be used as a threshold value $\sigma_{\text {noise }}$. This pedestal measurement was performed at the beginning of each data taking. After switching the X-ray beam on, the same detecting and readout cycle was repeated 100 to 1000 times. Two algorithms for detecting "X-ray signals" were then possible:

- using the RMS of the signal after subtracting the pedestal (analogue method) or

- counting events with a pedestal subtracted signal larger than $(3-4) \sigma_{\text {noise }}$ (binary method).

Typical signals for the analogue and binary method versus silicon strip channel number are shown in figures 8 and 9 , respectively. The former shows the signal in the $Z$-layer only, while the latter shows both the signals in $X$ and $Z$-layer. The signal in the in the $Z$-layer is wider due to the beam profile being small in $X$ and large in $Z$.

\section{Results}

\subsection{Properties of the Beam}

The X-ray counting rate $S_{X}=f(V, I, s, d)$ was analysed using the scintillation counter as described above, where $V$ is the high voltage at the anode of the $\mathrm{X}$ ray tube, $I$ the anode current, $s$ the width of the X-ray collimating slit, and $d$ the thickness of a filter (sheets of duraluminium, an aluminium alloy, were used) between scintillator and X-ray source.

Figure 10 shows the rate measured with the scintillator as a function of $I$ for $V=20 \mathrm{kV}, s=70 \mu \mathrm{m}$, and $d \approx 1 \mathrm{~mm}$. A linear behaviour is observed for rates below $3 \mathrm{MHz}$, as expected, while above a significant saturation is visible. This is caused by merging of the discriminated pulses from the scintillator occurring at high frequency due to a relatively small discriminator threshold. This curve is used to correct the data for this saturation effect.

Figure 11 shows the rate $S_{X}$ as a function of $V$ for $I=2 \mathrm{~mA}, s=70 \mu \mathrm{m}$, and $d \approx 1 \mathrm{~mm}$ after correcting for saturation. The solid curve is a fit according to the 
expected quadratic $V$-dependence:

$$
S_{X}=P_{1} \cdot\left(V-P_{2}\right)^{2}
$$

and shows good agreement. The parameter $P_{2}$ describes the offset due to the discriminator threshold.

Figure 12 shows the rate as a function of $s$ for $V=20 \mathrm{kV}, I=2 \mathrm{~mA}$, and $d \approx 1 \mathrm{~mm}$. An expected linear behaviour for low rates and the saturation effect at high rate, as described above, is observed.

To analyse the energy spectrum of the photons in the X-ray beam, the dependence of $S_{X}$ on $d$ for different voltages $V$ was analysed for $I=2 \mathrm{~mA}$ and $s=70 \mu \mathrm{m}$. The result corrected for saturation is shown in figure 13; the last two plots were derived from measurements with $s$ reduced to $20 \mu \mathrm{m}$ to limit the counting rate. The behaviour was modelled as follows: a raw X-ray spectrum according to equation (2) was assumed, allowing for an energy threshold due to the discriminator signal threshold. The intensity was attenuated according to equation (3) taking a small admixture of $\mathrm{Fe}, \mathrm{Mn}$ and $\mathrm{Cu}$ in the $\mathrm{Al}$ filter into account. The results, shown as curves in figure 13, are in good agreement with the measurements and confirm that the energy spectrum is described by equation (2).

The silicon detector was used to analyse the profile of the X-ray beam in $X$-direction using the counting rate as a function of channel number (ie strip position). The result is shown in figure 14. A narrow "core" and a broader "halo" is visible. The core is the part of the beam passing the collimation system via the "nominal" path. The halo is assumed to be caused by scattering within the collimator. Adding a filtering material (duraluminium) between the Si module and the beam reduces the ratio of halo to peak, showing that the halo is low energy X-ray quanta which would be consistent with being caused by scattering. A $1 \mathrm{~mm}$ thick filter is enough to absorb most of the halo.

\subsection{Predictions}

Equations (2) and (3) were used to determine the deposited intensity in silicon of thickness $300 \mu \mathrm{m}, I_{d}\left(E_{\gamma}\right)=I\left(E_{\gamma}\right)-I_{t r}\left(E_{\gamma}\right)$. To get a result corresponding to the different layers of the SCT, this was performed with different amount of absorber (zero, one, two, or three layers of $600 \mu \mathrm{m}$ silicon) in front of the detector-silicon, analysing different angles of inclination for both detector and absorber layers.

In order to determine the spectrum of intensity absorbed in a strip, it is essential to consider the case of a photon captured between two neighbouring strips generating a signal in both strips, and its energy split between them. So the observed intensity spectrum in a strip, $I_{c}\left(E_{\gamma}\right)$, will be "softer" than $I_{d}\left(E_{\gamma}\right)$. It was calculated from the simple assumption that the energy of such a photon splits equally between the two strips. It is further assumed the region where this occurs is of width $\delta$, with $\delta$ being equal to the range $s\left(E_{\gamma}\right)$ of the electron knocked 
out [8] as shown in figure 15. Thus the spectrum consists of the nominal part from outside the region $\delta$, contributing with a fraction $1-s\left(E_{\gamma}\right) / S$, and the split part, contributing with $s\left(2 E_{\gamma}\right) / S$ :

$$
I_{c}\left(E_{\gamma}\right)=I_{d}\left(E_{\gamma}\right) \cdot\left(1-s\left(E_{\gamma}\right) / S\right)+I_{d}\left(2 E_{\gamma}\right) \cdot s\left(2 E_{\gamma}\right) / S,
$$

where $S=50 \mu \mathrm{m}$ is the pitch of the strips in the silicon module.

The low energy part of the X-ray spectrum was ignored by only considering the intensity above an energy limit, $E_{\gamma}>10 \mathrm{keV}$. The fractional intensity $f$ for energies greater than a threshold value $E_{t r}$ is then defined as:

$$
f=\frac{\int_{E_{t r}}^{V} I_{c}\left(E_{\gamma}\right) d E_{\gamma}}{\int_{10 \mathrm{keV}}^{V} I\left(E_{\gamma}\right) d E_{\gamma}} .
$$

It is shown in figure 16. $E_{t r}$ is given in fC using the relation $E_{t r}[\mathrm{fC}]=E_{t r}[\mathrm{keV}]$. 0.044 , corresponding to a release of 276 electron-hole pairs per $\mathrm{keV}$ [1]. This allows a comparison with the electronics threshold used in the SCT readout. The parameter $V=40 \mathrm{kV}$, which is the working voltage of the X-ray tube, was assumed and the inclination of both detector and absorber layers was varied from $0^{\circ}$ to $45^{\circ}$.

The measurements described in section 4.1 allow the definition of the total rate, so that the absolute rate measured in a silicon strip in any layer of the $\mathrm{SCT}$ can be predicted. The total rate for typical beam parameters of $V=40 \mathrm{kV}$, $I=20 \mathrm{~mA}, s=70 \mu \mathrm{m}$ is $R_{0} \approx 23 \mathrm{MHz}$. From geometrical considerations only approximately the fraction $S / c=0.57$ of the rate is seen, where $S$ is the pitch of the strips and $c$ is defined by equation (1). Figure 17(top) shows the captured total rate in a strip for an amount of absorbing Si corresponding to the first and fourth SCT layer with an inclination ${ }^{4}$ of $20^{\circ}$ as functions of $E_{t r}$. Overlayed is the noise level of a Si module derived from test beam measurements [9] or as assumed for Monte Carlo simulations [1]. The bottom plot in figure 17 shows the signal to noise ratio $S / N$ for an amount of absorbing Si corresponding to the first and last layer of the SCT, assuming the higher noise estimate. Obviously a threshold defining a sufficiently high $S / N$ can be found.

\subsection{Position Determination}

\subsubsection{Strip Weighting Method}

The very precise pitch of the silicon strips themselves can be used to reconstruct the position of the silicon module. If only one strip is hit, the accuracy of the positioning will be equal to $50 \mu \mathrm{m} / \sqrt{12}=14 \mu \mathrm{m}$. But as the width of the beam can be chosen to be large enough to produce a signal in two or more strips, a

\footnotetext{
${ }^{4}$ An inclination of $15^{\circ}$ to $30^{\circ}$ is expected from geometrical considerations for a scan of the SCT.
} 
weighting of the position of the strips according to their counting rates yields a much better accuracy. Figure 20 shows the counts for 1000 measurements (binary method) versus channel number for an X-ray beam with $V=40 \mathrm{kV}, I=10 \mathrm{~mA}$ and $s=15 \mu \mathrm{m}$ for four measurements with the beam position shifted by the linear stage by $10 \mu \mathrm{m}$ each. The curve shows the result from a fit with the sum of a narrow and a wide Gaussians and a constant, representing the beam core, the halo and noise, respectively. The observed width of the peak is somewhat larger than the width of the beam profile ( $c \approx 20 \mu \mathrm{m}$ from equation (1) for $s=15 \mu \mathrm{m}$ ) which is expected from the data acquisition method enhancing the rate in the tails.

To estimate the achievable accuracy the X-ray beam position was measured by the Si module with above fitting method and simultaneously by the linear stage changing the distance in steps of $10 \mu \mathrm{m}$, see figure 21(left). The relation between both measurements was fitted by a straight line, and the residual distribution for the Si data is shown in the right plot of figure 21. The RMS defines the repeatability which is better than $5 \mu \mathrm{m}$. A wider X-ray beam provides an even more accurate positioning, see figure 22 .

\subsubsection{Scanning Method}

Another possible method of position reconstruction is the measurement of the $\mathrm{X}$-ray rate as a function of the scanner position. For this purpose, a certain strip of the detector was selected and the number of X-ray counts for a total of 100 measurements (binary method) was recorded as a function of the position of the linear stage for an X-ray beam with $V=40 \mathrm{kV}, I=10 \mathrm{~mA}$ and $s=30 \mu \mathrm{m}$. An example of a typical rate-versus-position plot is given in figure 18. The rate was fitted with a convolution of a rectangular function and a Gaussian, representing the shape of a strip and the beam profile, respectively.

From these fits the central position was determined and compared to the nominal position from the linear stage. In addition, the difference in the fitted positions of two successive scans was analysed as shown in figure 19. The width of these distributions give a measure of the repeatability of the measurements and thus their precision, which is approximately $3 \mu \mathrm{m}$.

The advantage of this method is its small sensitivity to noise and that the efficiency of only one strip is involved. Its disadvantage is that the scanning requires movement of the X-ray beam so that the time needed is larger than in the previous method.

\subsubsection{Influence of Absorbing Material}

The intensity profiles measured by the Si module was analysed for different amount of duraluminium (from $25 \mu \mathrm{m}$ to $7.6 \mathrm{~mm}$, corresponding to up to $6 \mathrm{~mm}$ silicon for absorption of $40 \mathrm{keV}$ photons) placed between Si detector and X-ray 
source. Figure 23(left) shows the change in reconstructed position as a function of absorber thickness. The RMS of $2 \mu \mathrm{m}$ shown in the right plot demonstrates that the position reconstruction is essentially insensitive to uniform absorbing material.

The possible effect of artificial patterns in the rate due to absorption in the support material (carbon fibre honeycomb) was tested measuring the rate in the scintillation counter while moving a sample of this honeycomb across the X-ray beam. The result shown in figure 24 for different inclination angles of the material with respect to the beam shows at most a dip of $10 \%$ in the rate, being smeared for increasing inclination. To estimate the effect on the reconstruction of the position of silicon detectors, a simple check was performed as follows. The dip as measured with the rate scans was parametrised. The parametrisation was used to modify a Gaussian with sigma corresponding to the beam width, shifting the relative position. The result was fitted with a Gaussian and the fitted central position was compared with the original one. A typical example of this is shown in figure 25(top). Taking the maximum difference in position gives a measure of the "worst case" error due to absorption in the honeycomb. It was analysed as a function of beam width (ie the Gaussian sigma) as shown in figure 25(bottom). The result demonstrates that the effect can be kept small if the width of the beam is chosen to be small enough.

\subsubsection{Tilted Si Module}

The nominal scans above were performed with the silicon detector being in the $X Z$-plane. However, measurements with detectors in the SCT will involve an inclination of the detector with respect to the X-ray beam axis. The rate scans described above were thus repeated with a detector inclination of $30^{\circ}$ with respect to the $X Z$-plane to check effects on the resolution of the position reconstruction. The rates demonstrate an increased width of the beam profile as expected from geometrical considerations while the resolution in the reconstructed position shows only slight differences to the above result, see figure 26 . A more significant change is only visible if one increases the inclination to $45^{\circ}$ as shown in figure 27 .

\subsubsection{Rotated Si Module}

For the nominal measurements above the strips of the silicon detector in $X$ direction were adjusted to be parallel to the beam profile. As this is not necessarily true for real measurements, the effect of the silicon detector being rotated in the $X Z$-plane was checked. For this purpose the rate scans described above were repeated with the detector rotated by $1.3^{\circ}$. The rate profile with rotation becomes wider as expected, see figure 28 . The resolution of the position reconstruction is, however, not affected as shown in figure 29 . 


\subsubsection{Bias Voltage}

For completeness, the dependence of the signal of the X-ray beam on the bias voltage $U_{\text {bias }}$ of the silicon detector used was measured. Scans similar to those described above were performed for $U_{\text {bias }}$ from 10 to $80 \mathrm{~V}$, determining the peak efficiency (number of counts in the peak for a total of 100 counts), the fitted position and half-width. This was considered for a detector inclination of $0^{\circ}, 30^{\circ}$ and $45^{\circ}$. The results are shown in figures 30,31 and 32 in the above order of inclination. They demonstrate:

- an increasing efficiency with increasing $U_{\text {bias }}$, which is smaller for larger inclination angle

- a negligible $\left(0^{\circ}\right)$ to significant $\left(45^{\circ}\right)$ dependence of the position on $U_{\text {bias }}$;

- an increase in the width of the signal, which is larger for large inclination angles.

From these observation it seems to be important to ensure a complete depletion which is given for $U_{\text {bias }}>60 \mathrm{~V}$. As a compromise with noise levels rising with $U_{\text {bias }}$ a bias voltage of $60 \mathrm{~V}$ was chosen.

\subsubsection{Wire and Pin Shadow Method}

To determine internal parameters of the planned two and three dimensional scanners, metrologically precisely positioned objects have to be measured by scanning. For this purpose, passive targets like wires or pins will be used. The precision of scanning of such objects was tested.

The first test was performed with a $50 \mu \mathrm{m} \mathrm{W}$ wire as target. For this purpose a wire was installed approximately in $Z$ direction on the linear stage and moved in $10 \mu \mathrm{m}$ steps in $X$ direction between X-ray and scintillator. The "shadow" of the wire was observed as a sharp dip in the scintillator rate (see top plot in figure 33). A fit with a constant minus a Gaussian in the dip region yielded good results, with the Gaussian mean giving the wire position. The resolution in the difference between the positions of two successive scans is $1 \mu \mathrm{m}$ as shown in figure 33(bottom), which is the precision of the linear stage. The repeatability of this position determination is thus better than $1 \mu \mathrm{m}$.

Another test was performed with a $1.5 \mathrm{~mm}$ steel pin as target similarly to the scan of a $\mathrm{W}$ wire described above. The falling or rising part of the rate-versusposition plots were fitted with a linear behaviour (see top plot in figure 34). Together with the minimum and the maximum rate this yields the position of the half-width. Combining the half-width position from both sides defines the centre position. The repeatability of this procedure was again found to be better than $1 \mu \mathrm{m}$ as shown in figure 34 (bottom). 


\section{Conclusion}

First measurements with a 1D X-ray scanning facility have produced encouraging results. In particular the reconstruction of the position of silicon modules was performed with an accuracy of a few microns. This result was essentially stable when the general conditions (absorber, inclination, rotation) and internal parameters were changed. Simulations based on measurements of beam properties showed that the signal rate in all four layers of the SCT will be sufficient to perform a survey with the present X-ray beam setup.

It was shown that the scanning of passive objects has a precision of better than $1 \mu \mathrm{m}$ so that the calibration of internal parameters of a more complicated X-ray system can be based on this.

The next phase in developing the SCT survey will be the construction of a 2D scanning facility with a rotary stage.

It should be stated here that for measuring the real SCT multilayer structure the time interval $\Delta t \approx 10 \mu$ for a data capture cycle, ie $t_{\mathrm{DAQ}} \approx 10 \mathrm{~ms}$ per point will be enough. In addition, time is needed to move the rotation head from one point to another. It was measured ${ }^{5}$ to $t_{\text {rot }} \approx 400 \mathrm{~ms}$. Three or four points define the position of every separate silicon module. Thus the time for measuring a module will be $t_{\mathrm{mod}}=4 \cdot\left(t_{\mathrm{DAQ}}+t_{\mathrm{rot}}\right) \approx 1640 \mathrm{~ms}$, and the time for measuring a whole circle with approximately 50 modules will be $t_{\text {circ }}=50 \cdot t_{\bmod }=82 \mathrm{~s}$. Adding the time for turning the rotation head back by $360^{\circ}$ and moving in longitudinal direction for the next circle (approx. $50 \mathrm{~s}$ ), the coordinate calibration of one barrel of the SCT will take 4 layers $\times 40$ circles $\times 132 \mathrm{~s} \approx 21000 \mathrm{~s}$, the so called live time. The real time, 10 times larger (58 hours or $2 \frac{1}{2}$ days), still seems reasonable.

\section{Acknowledgements}

The helpful and competent assistance of P.Pattinson in all our technical problems was invaluable. We appreciated the advice of G.L.Salmon in the selection of scintillator material and phototubes.

\section{References}

[1] ATLAS Technical Design Report - Inner Detector, CERN/LHCC/97-16 and CERN/LHCC/97-17 (1997).

[2] L.Vertogradov, High precision wire' positions measuring in the drift tube package by the X-ray scanner (XTomograph), CERN, ATLAS Internal Note, MUON-NO-041 (1994).

\footnotetext{
${ }^{5}$ An ADR200-11-RE10 rotation stage from AEROTECH, Pittsburgh was equipped with an $\mathrm{X}$-ray tube similar to the one used here to perform these checks.
} 
O.L.Fedin and A.I.Smirnov, On a possibility to use a narrow X-ray beam for straw proportional tubes' alignment, CERN RD-6 Note 51 (1994).

I.P.Boyko et al., Space positions measuring of the signal wires inside drift tubes by help of X-ray beam (in Russian), Short Messages of JINR 2[70]-95, Dubna (1995).

[3] SVETLANA Electron Device Mfg.Corp., Catalogue: X-ray Tubes, Portable X-ray Units (1995) 27.

[4] Linear stage and PC23 controller documentation from: Naples Coombe Ltd., Main Street, Chaddleworth, Berkshire, England.

[5] V.Chabaud et al., The DELPHI Silicon Strip Microvertex Detector with Double Sided Readout, Nucl. Instrum. Methods A368 (1996) 314.

[6] see eg: Encyclopedia of Physics: X-Rays, S.Flügge (editor), Springer, Vol. 30 (1957).

[7] J.H.Hubbell, Photon Cross Sections, Attenuation Coefficients, and Energy Absorption Coefficients From $10 \mathrm{eV}$ to $100 \mathrm{MeV}$, Int. J. of Appl. Rad. \& Isotopes 33 (1982) 1269.

[8] S.T.Perkins, D.E.Cullen, and S.M.Seltzer, Tables and Graphs of Electron Interaction Cross Sections from $10 \mathrm{eV}$ to $100 \mathrm{MeV}$, UCRL-50400, Vol. 31 (1991).

[9] A.Grewal, thesis in preparation. 


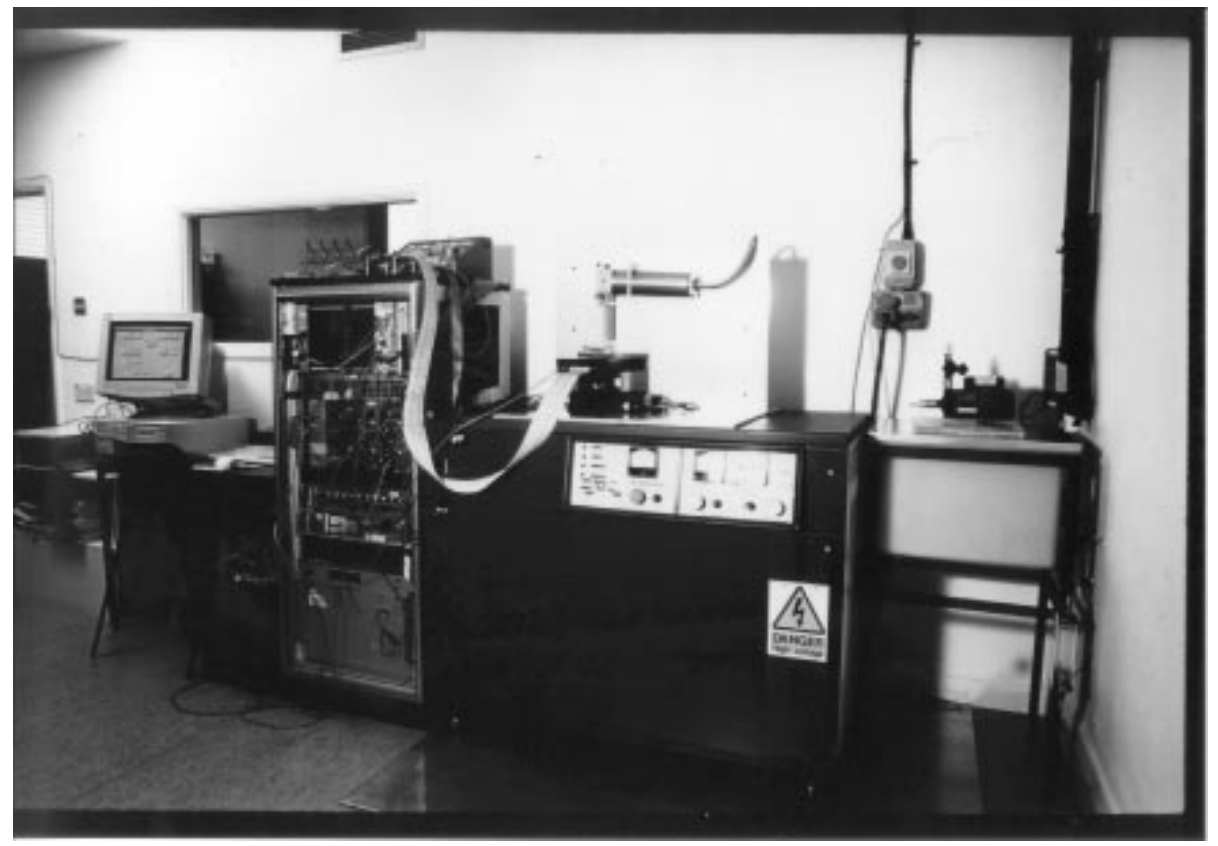

Figure 1: Photo of a general view. From left to right: PC, rack with DAQ electronics, X-ray scanning facility on top of the high voltage power supply.

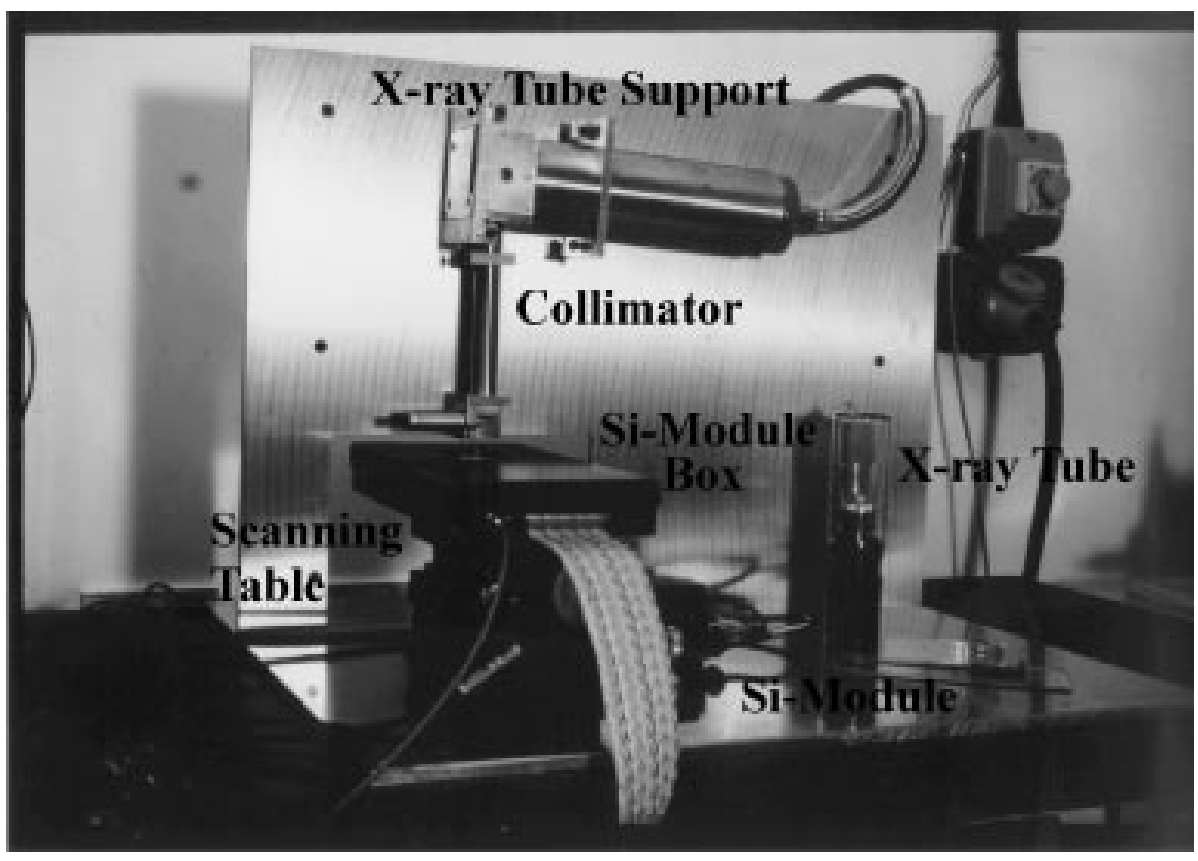

Figure 2: Photo of the 1D X-ray scanning facility: X-ray tube support and socket, collimator, Si-module box, $X Z$ scanning table. Separately the X-ray tube and the silicon module are shown. 

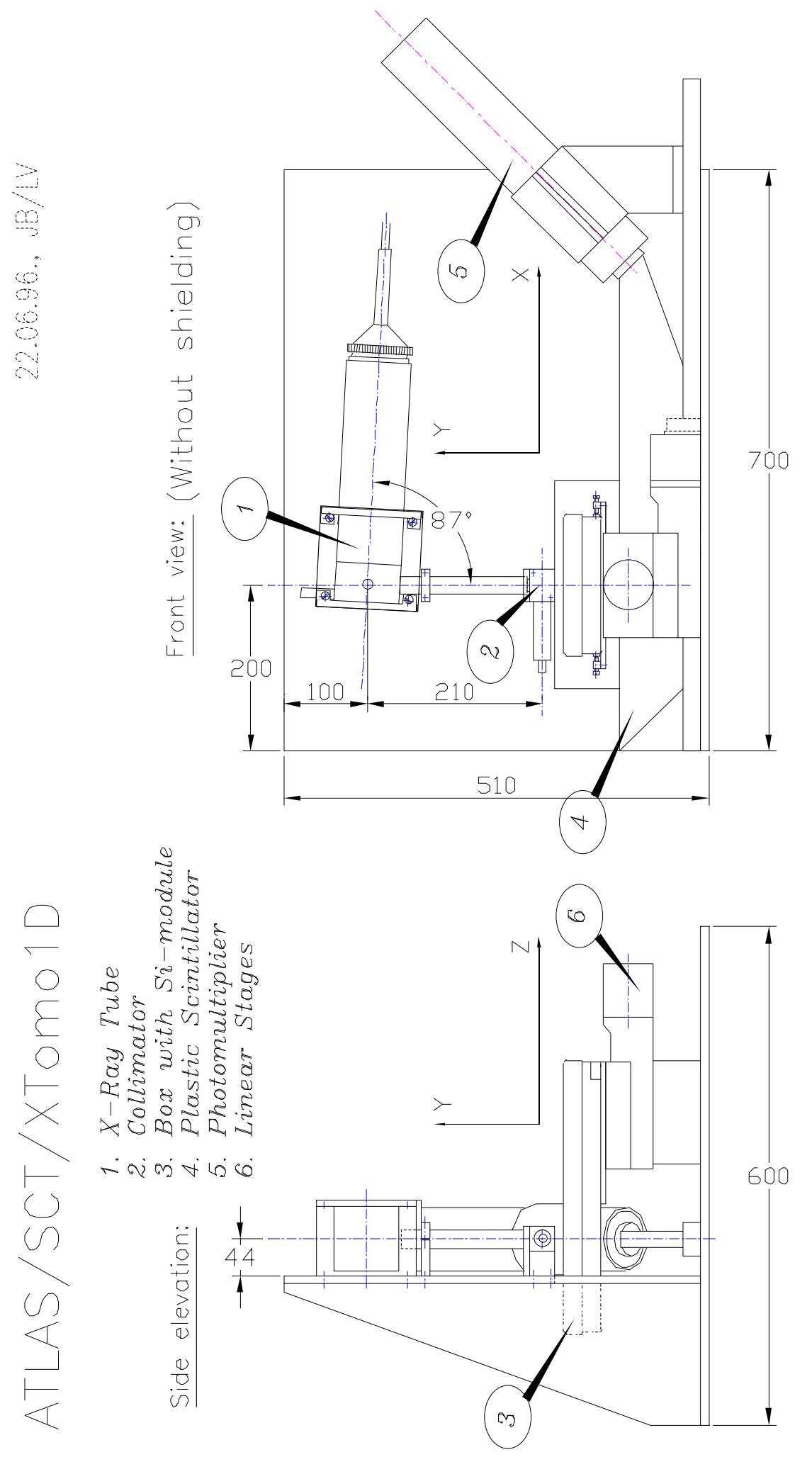

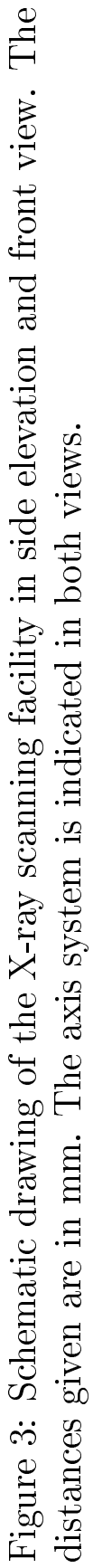




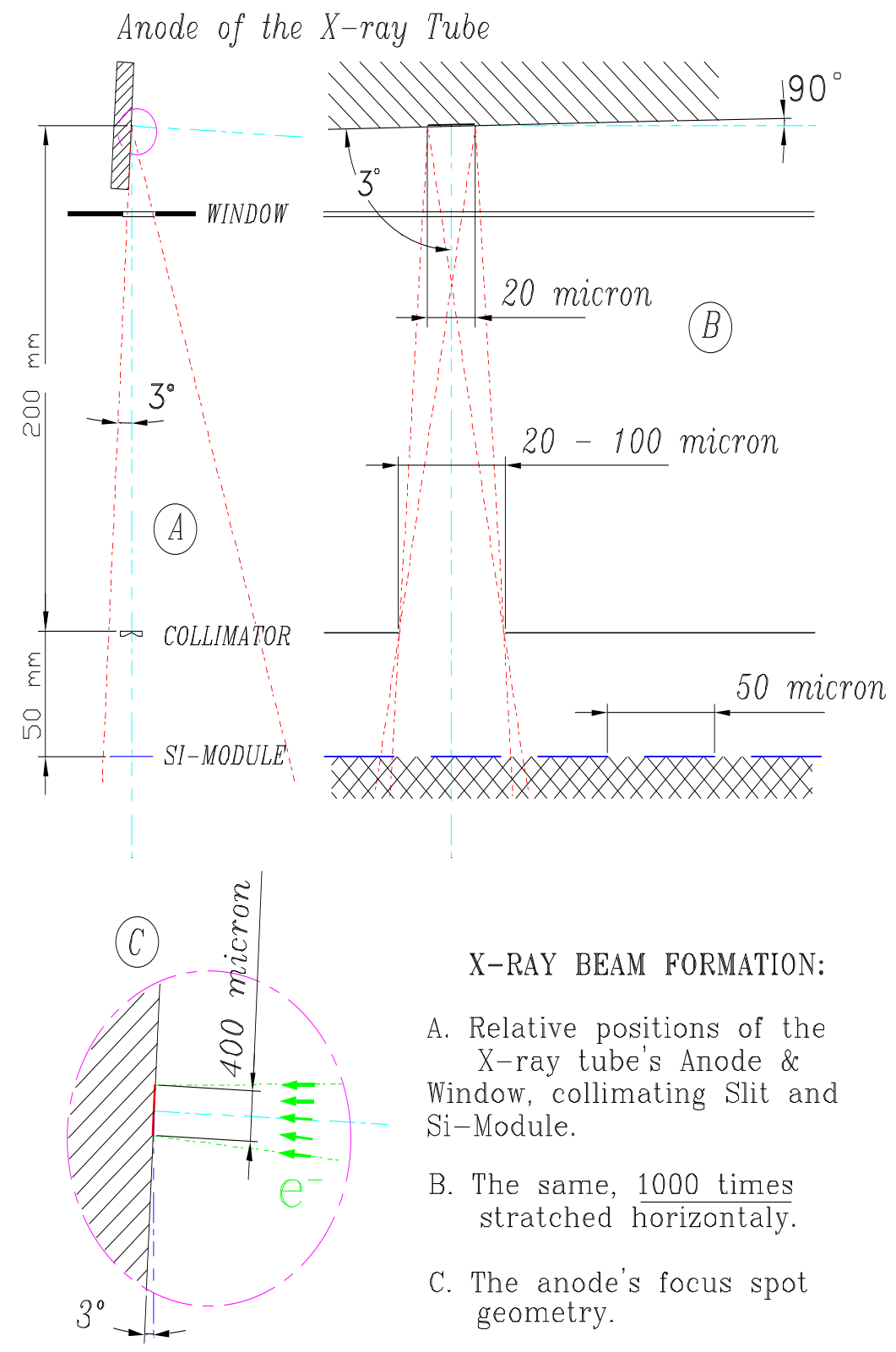

Figure 4: X-ray beam formation diagrams: (a) the collimating slit position relative to the anode of the tube; (b) the same picture 1000 times scaled along the $X$ axis only; (c) anode's focus spot. 

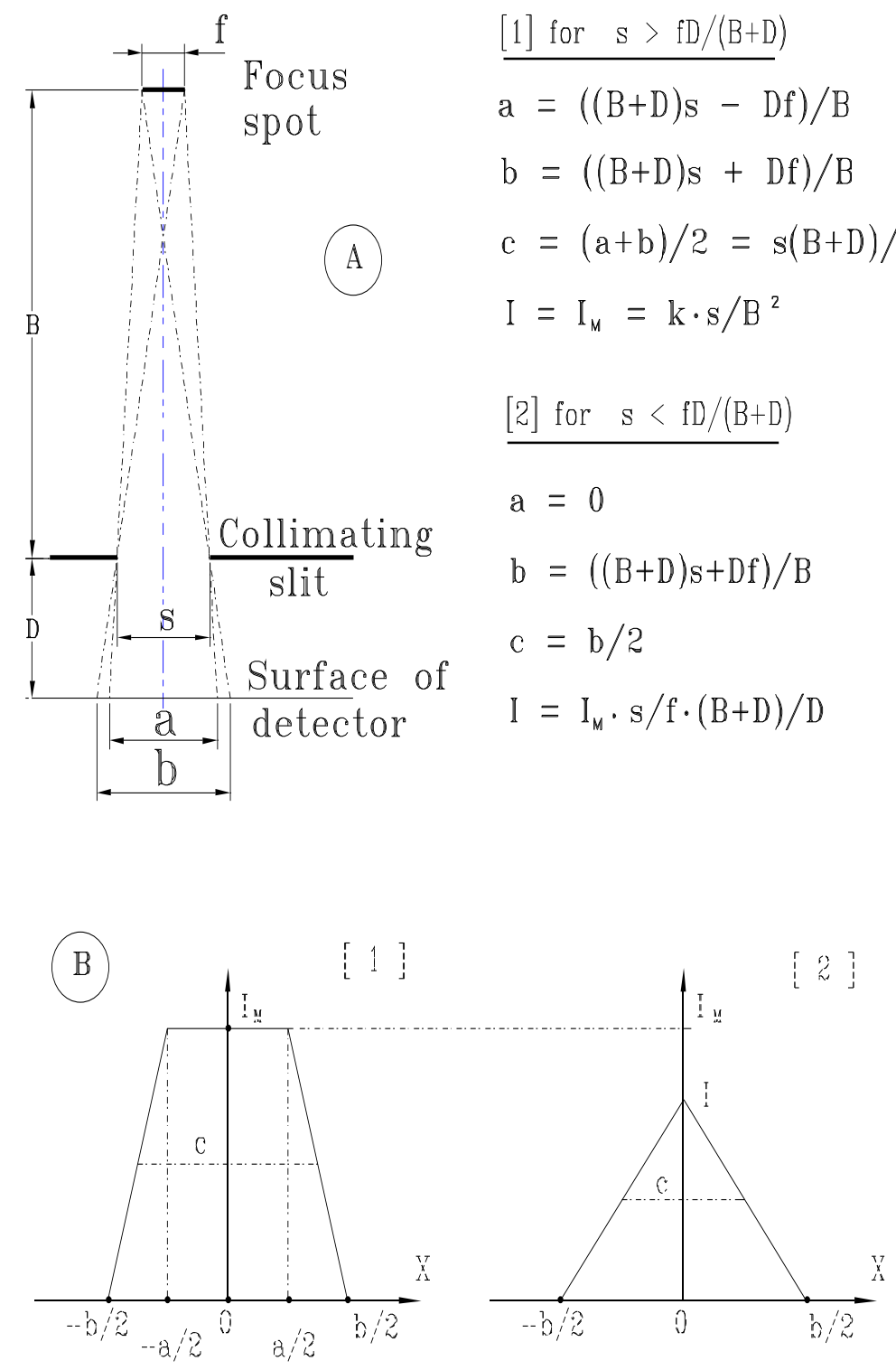

Figure 5: Simplified geometrical calculations of the X-ray beam intensity profile: (a) beam collimating diagram and formula; (b) two types of the profile shapes. 


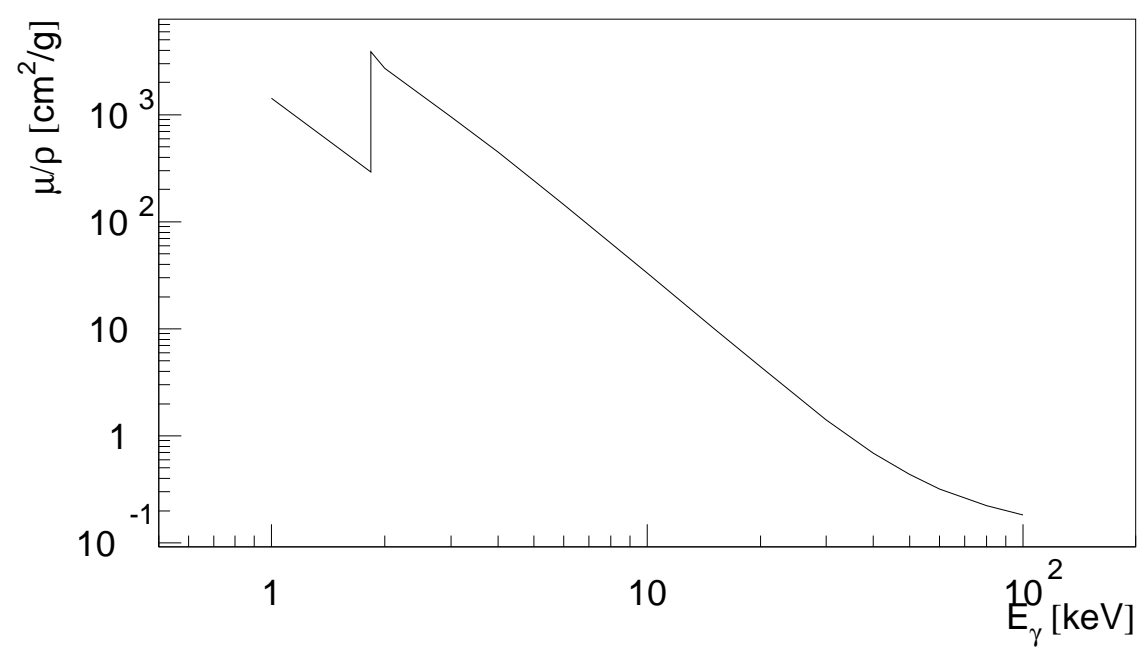

Figure 6: Attenuation mass coefficient $\mu$ in silicon normalised to the density $\rho$ as a function of the X-ray photon energy $E_{\gamma}$.

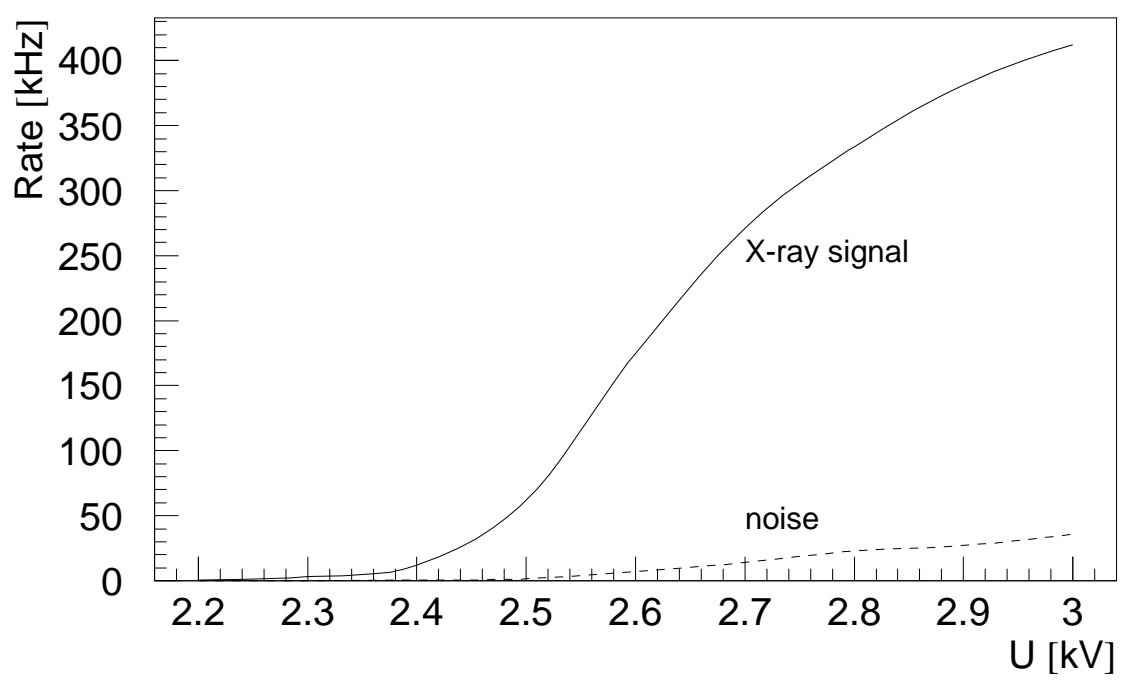

Figure 7: Noise and signal rate for the scintillation counter as a function of the voltage $U$. 


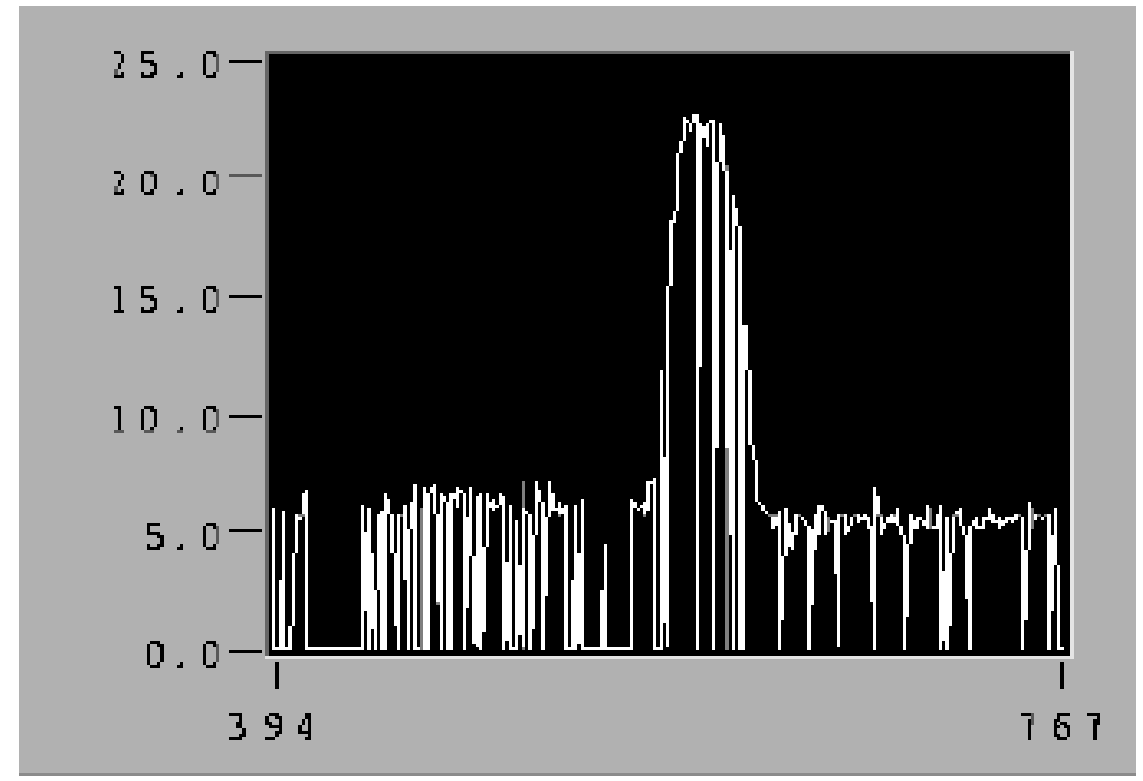

Figure 8: RMS of silicon signals versus channel number showing a clear peak at the X-ray penetrating position ( $Z$-layer only).

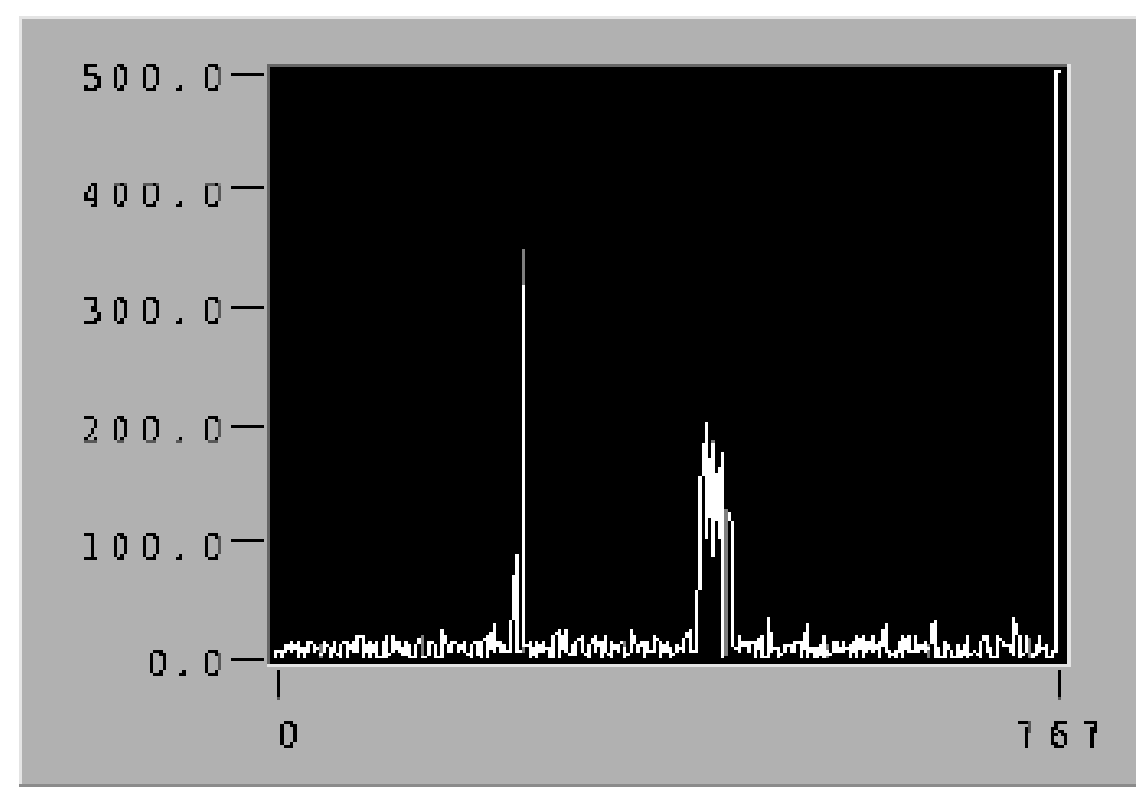

Figure 9: Counting rate in silicon strips derived as explained in the text versus channel number. Clear peaks from the X-ray signal in the $X$ (left) and $Z$ (right) layer of the module are visible. 


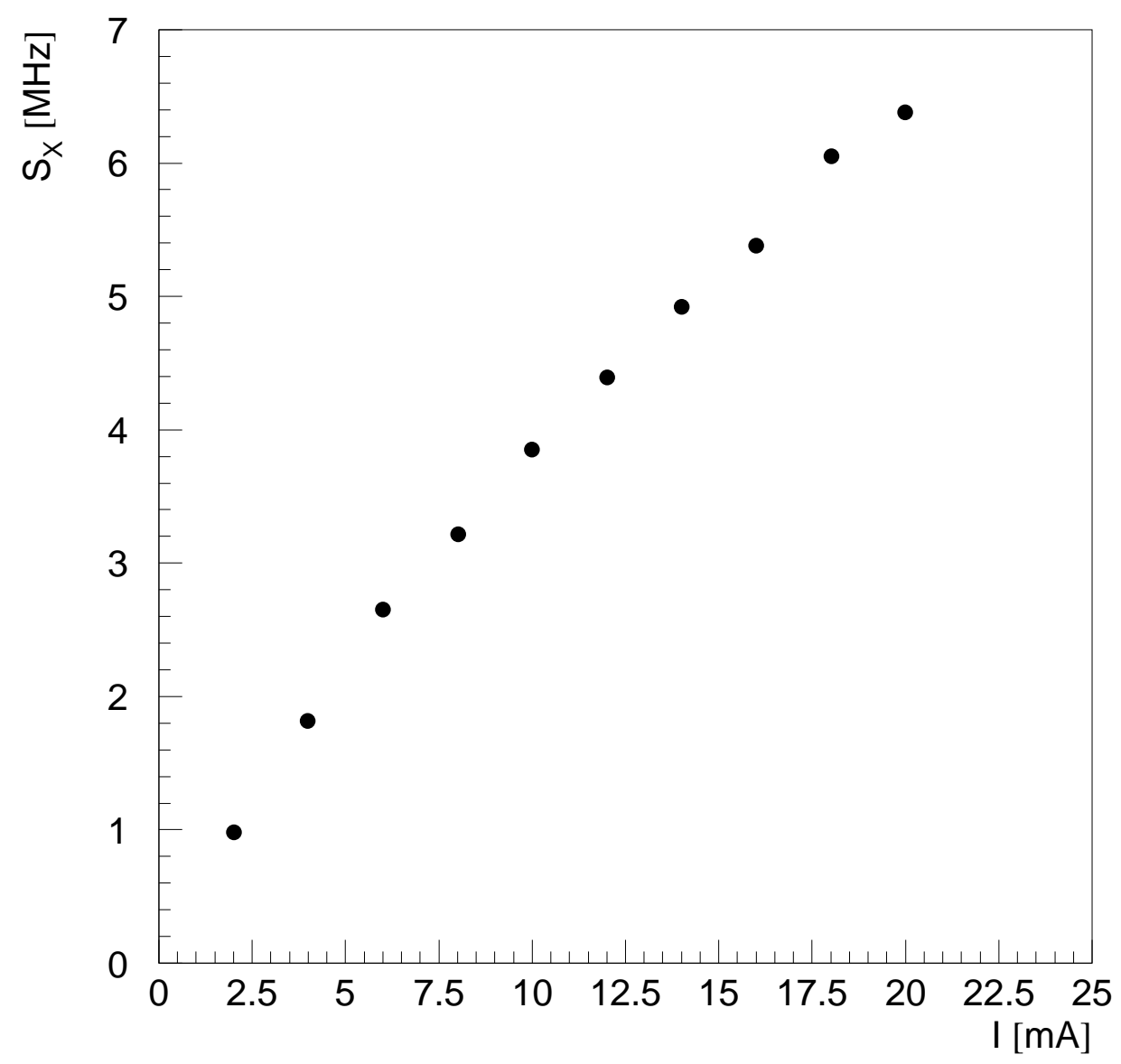

Figure 10: X-ray counting rate $S_{X}$ measured in the scintillator as a function of the X-ray tube anode current $I$. 


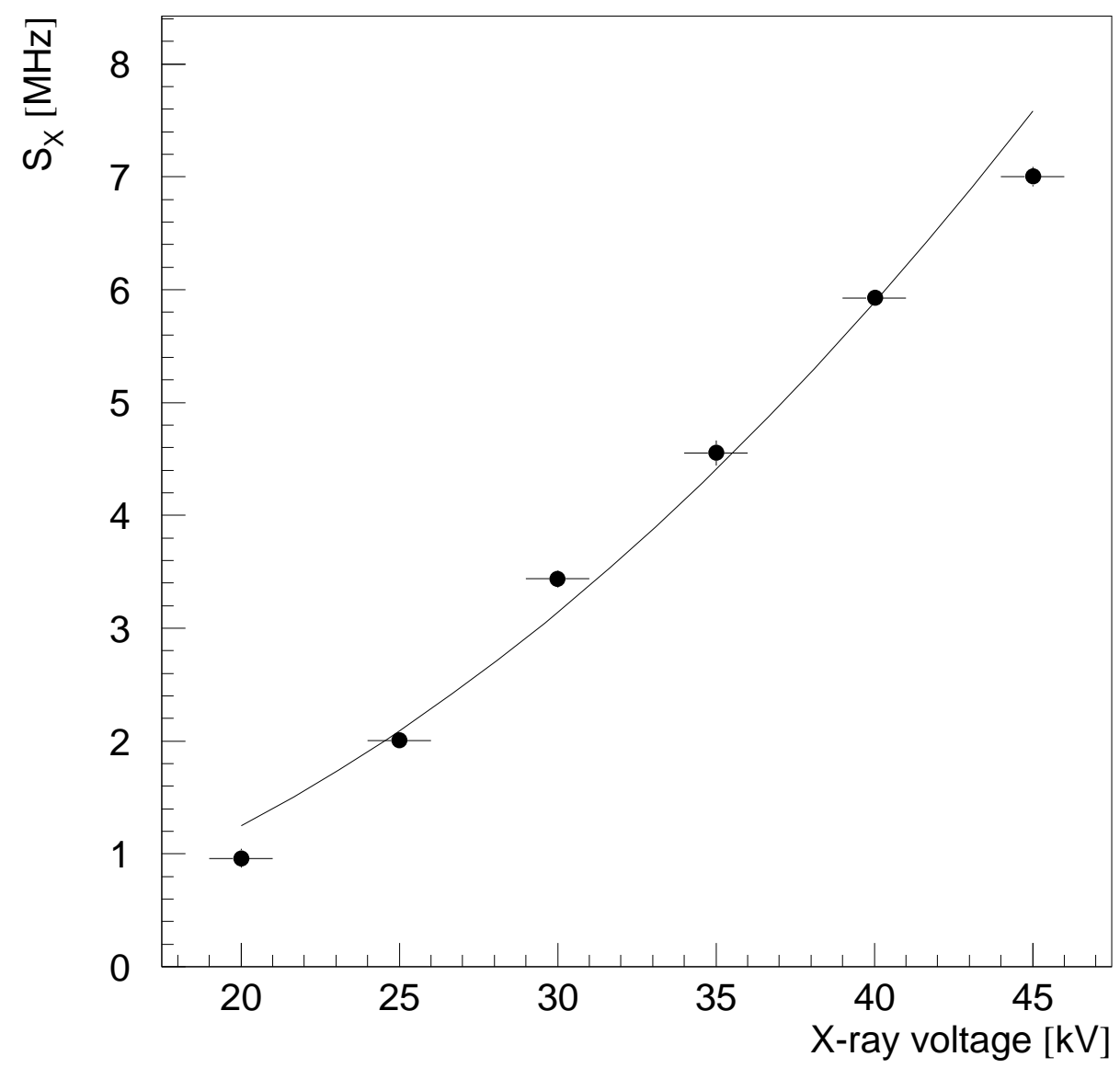

Figure 11: X-ray counting rate $S_{X}$ measured in the scintillator as a function of the X-ray tube anode high voltage $V$. The curve represents the result from a fit with a parabola. 


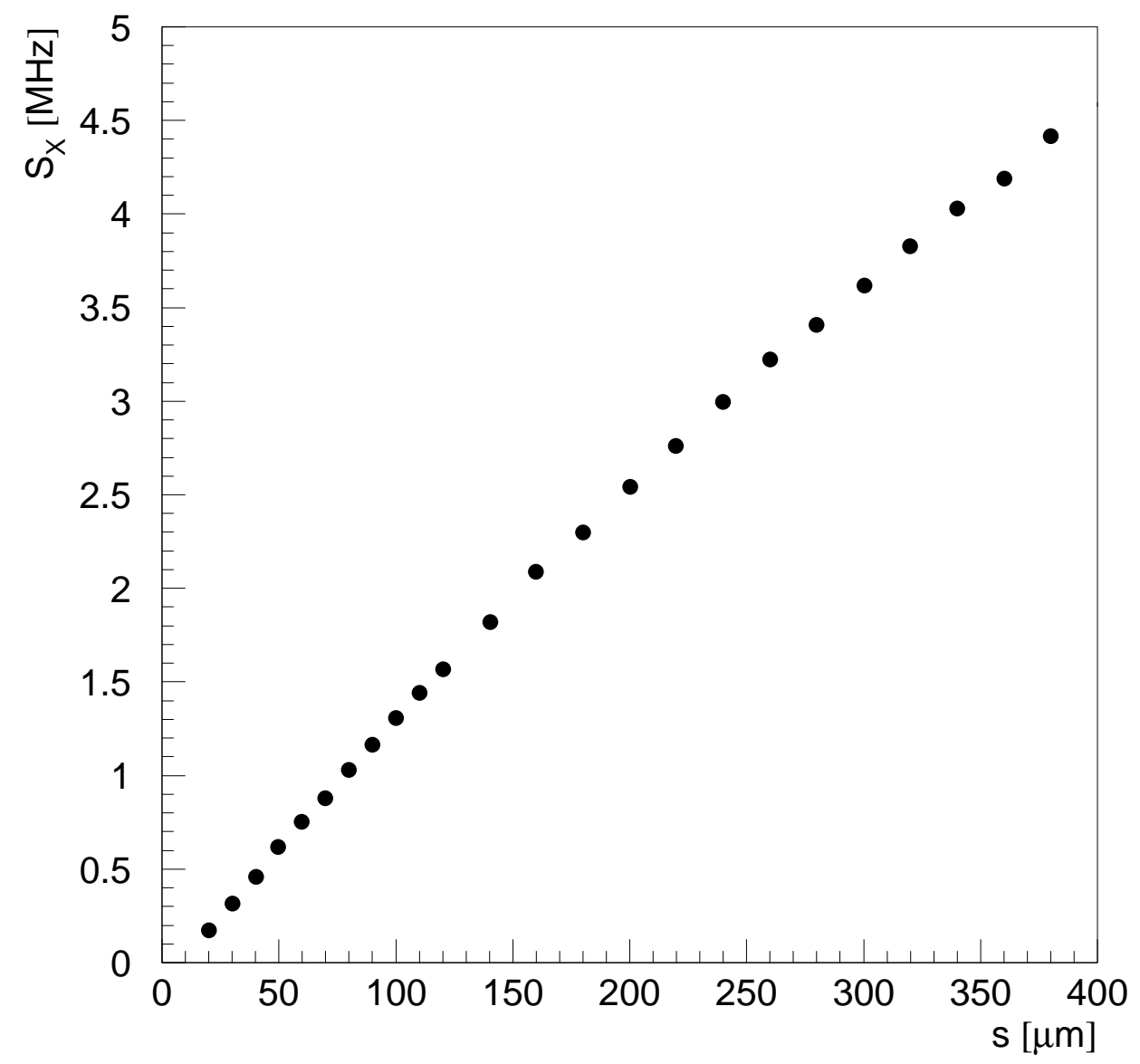

Figure 12: X-ray counting rate $S_{X}$ measured in the scintillator as a function of the collimator slit width $s$. 

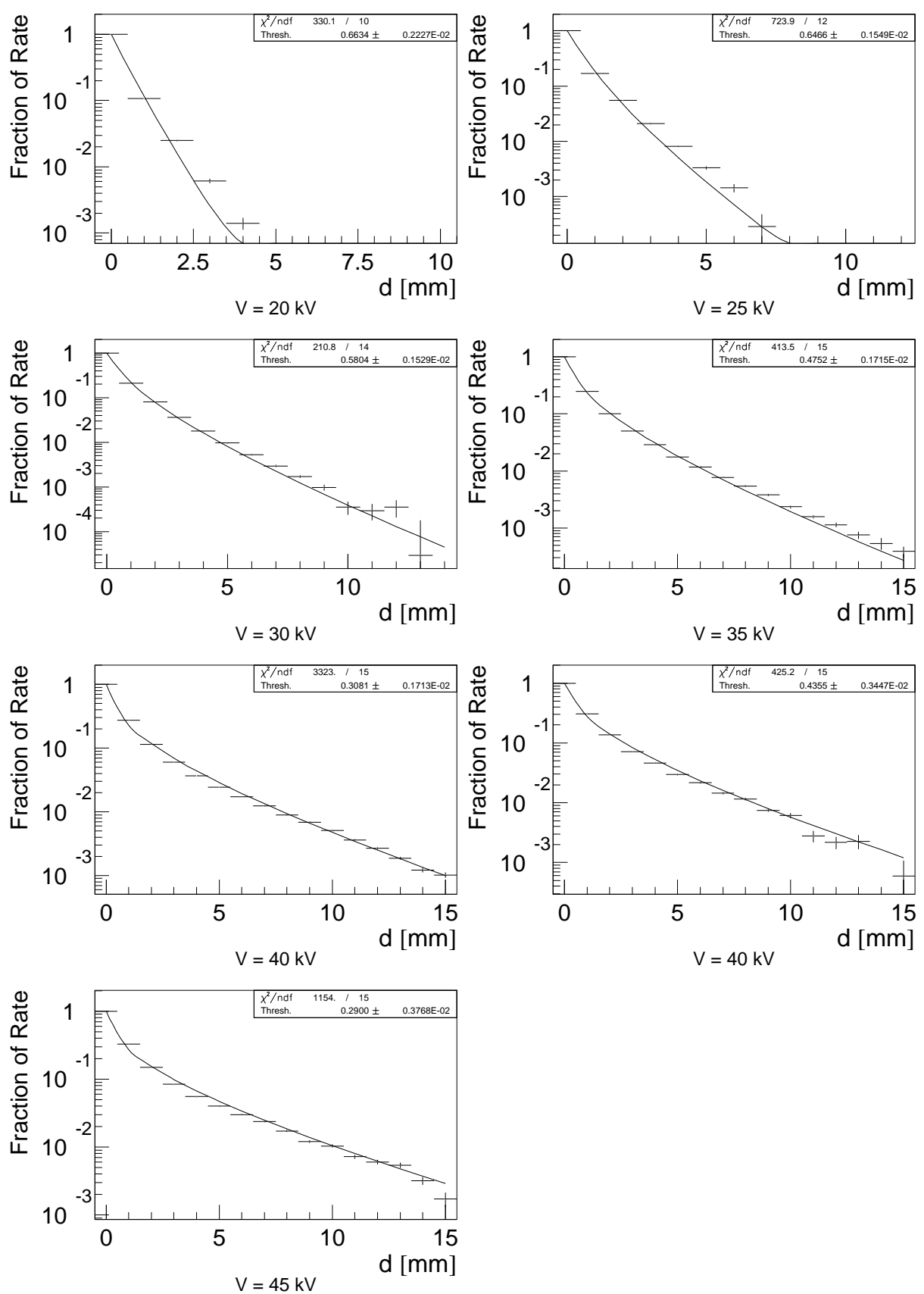

Figure 13: Normalised counting rate $S_{X} / S_{X}(d=0)$ measured in the scintillator as a function of the thickness $d$ of a duraluminium absorber between X-ray and scintillator for different anode high voltage $V$. The curves show the results of fits explained in the text. The last two plots were derived from measurements with smaller collimator slit width $s$ (to limit the rate). 

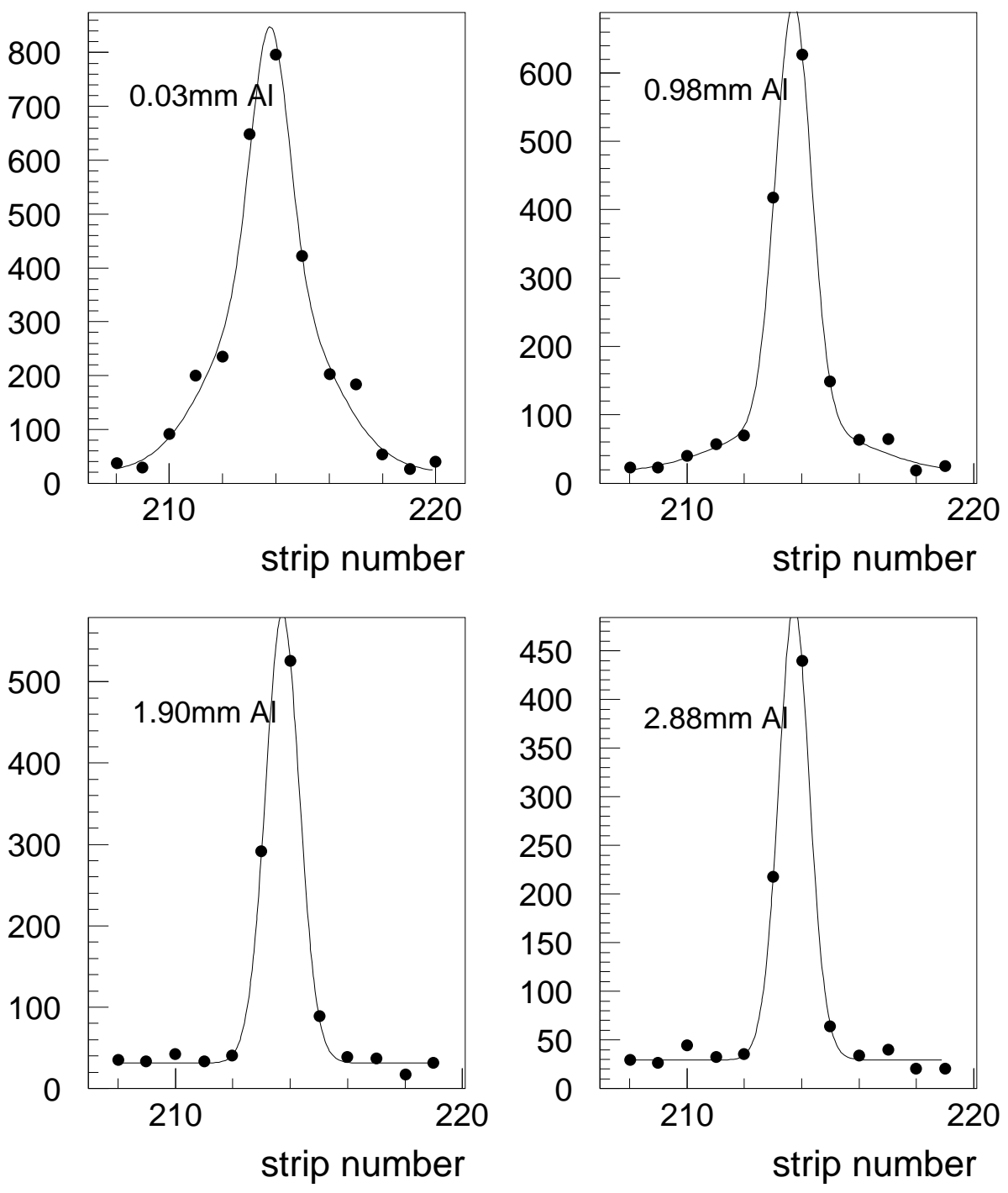

Figure 14: Sequential measurements of the rate in the Si-module as a function of channel number at the same position of the X-ray beam. Adding duraluminiumfilters (the total thickness is given in each plot) demonstrates the change in the beam shape and the removal of the halo. 


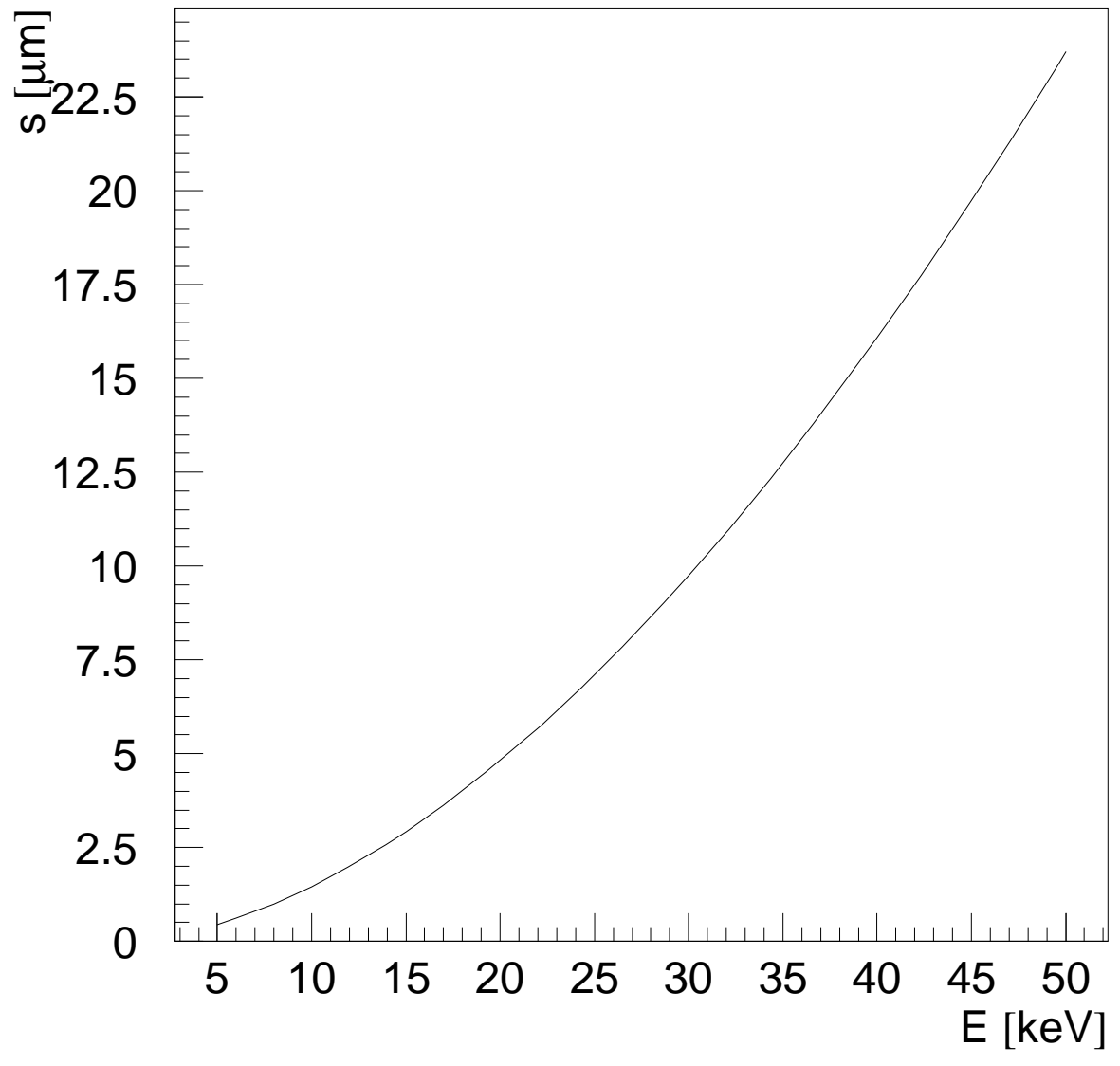

Figure 15: Free range $s(E)$ of an electron in silicon as function of its energy $E$. 


\section{X-ray Signal $(E>10 \mathrm{keV})$ in $300 \mu \mathrm{m} \mathrm{Si}$}
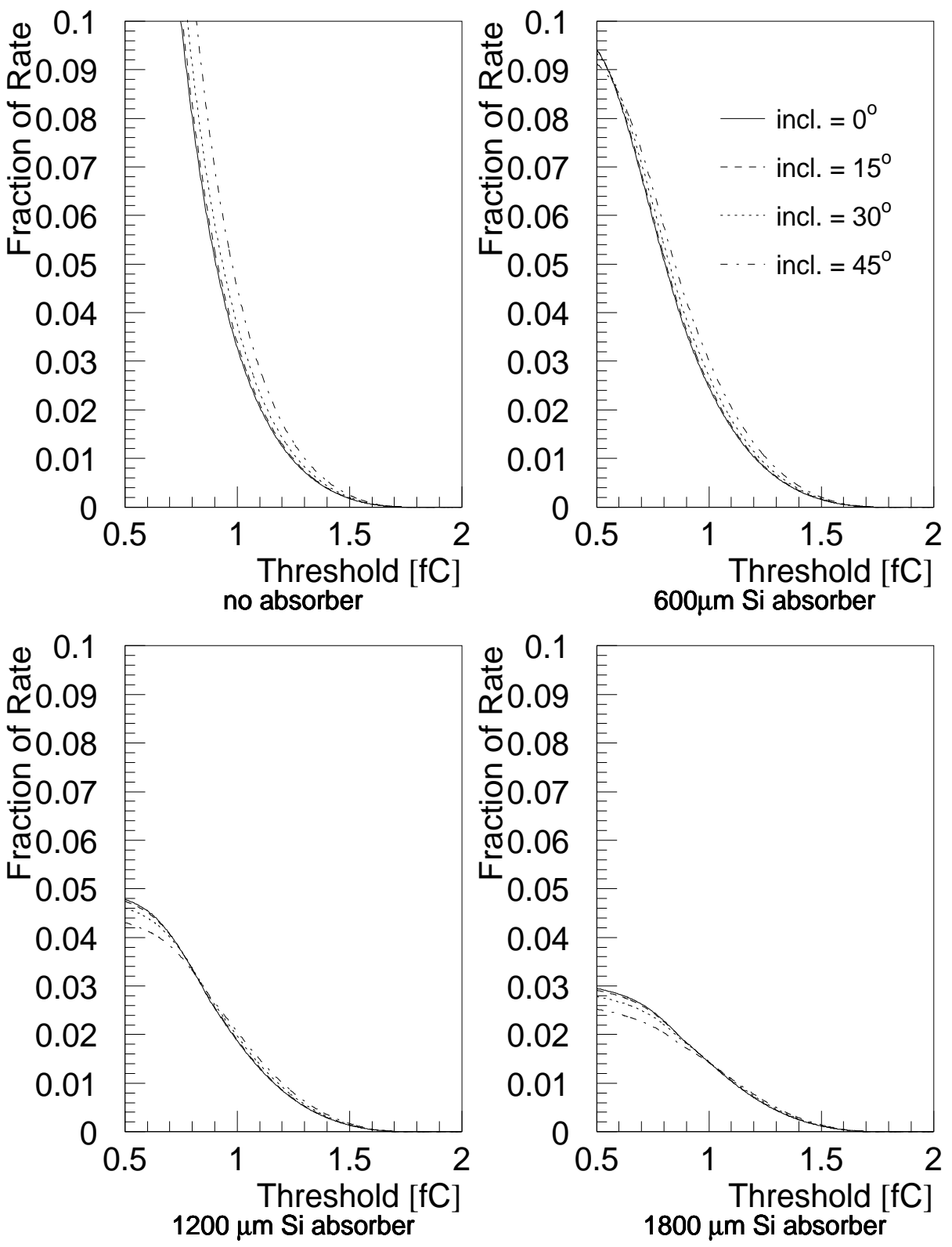

Figure 16: X-ray intensity spectrum normalised to the total rate for $E_{\gamma}>10 \mathrm{keV}$ as a function of the readout threshold calculated for a $300 \mu \mathrm{m}$ thick Si module after absorption in zero to three layers of $600 \mu \mathrm{m}$ Si. Different angles of inclination of both detector and absorber and a high voltage of $V=40 \mathrm{kV}$ were assumed. 

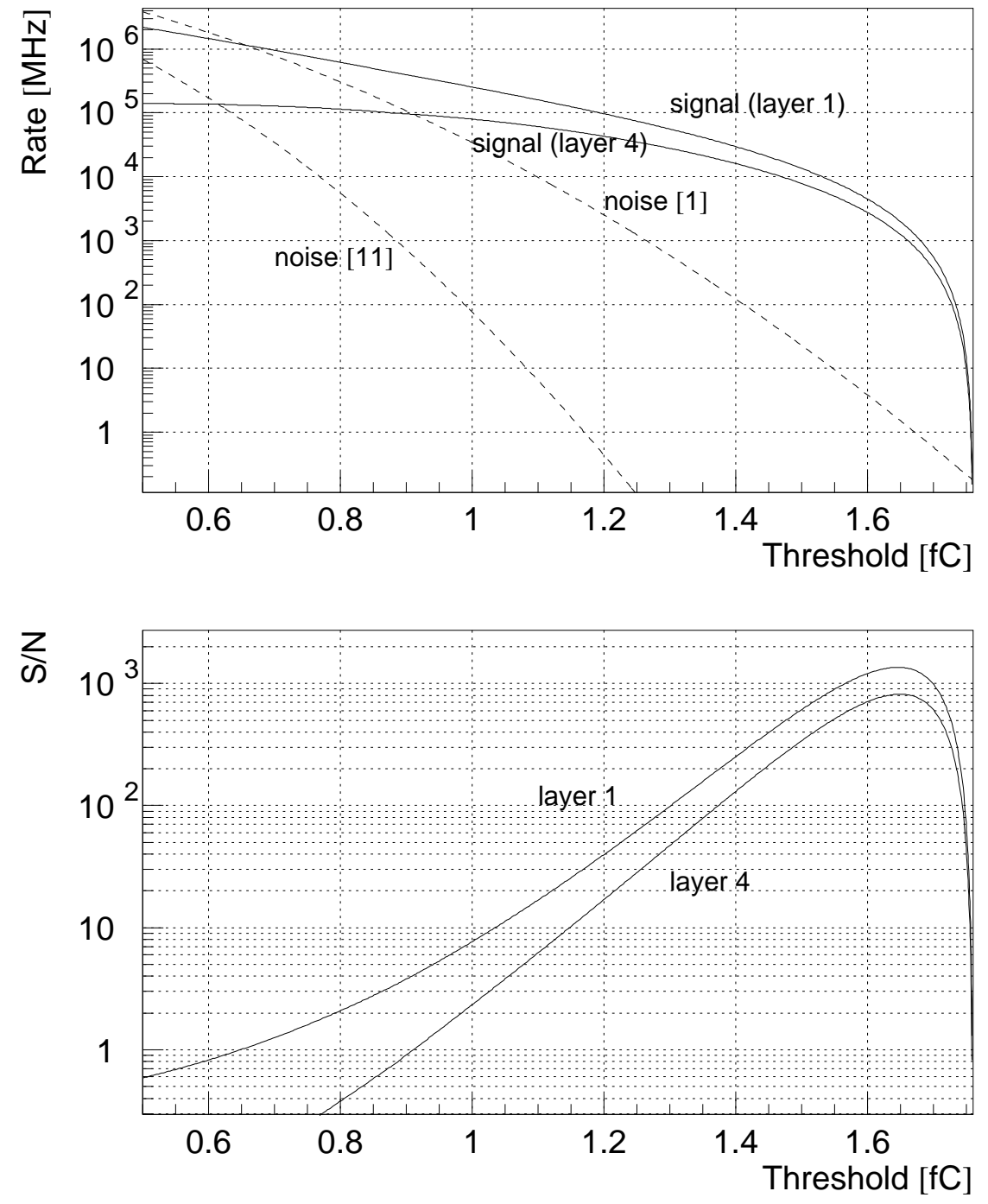

Figure 17: Top: estimated X-ray rate in the first and fourth layer of the SCT for $V=40 \mathrm{kV}, I=20 \mathrm{~mA}$, and $s=70 \mu \mathrm{m}$ (solid lines) compared with the noise level assumed in [1] and measured in [9] (dashed lines) as function of the readout threshold. Bottom: signal to noise ratio $S / N$ for first and fourth SCT layer, assuming the noise estimate from [1]. 


\section{Scanning of Si Strip}
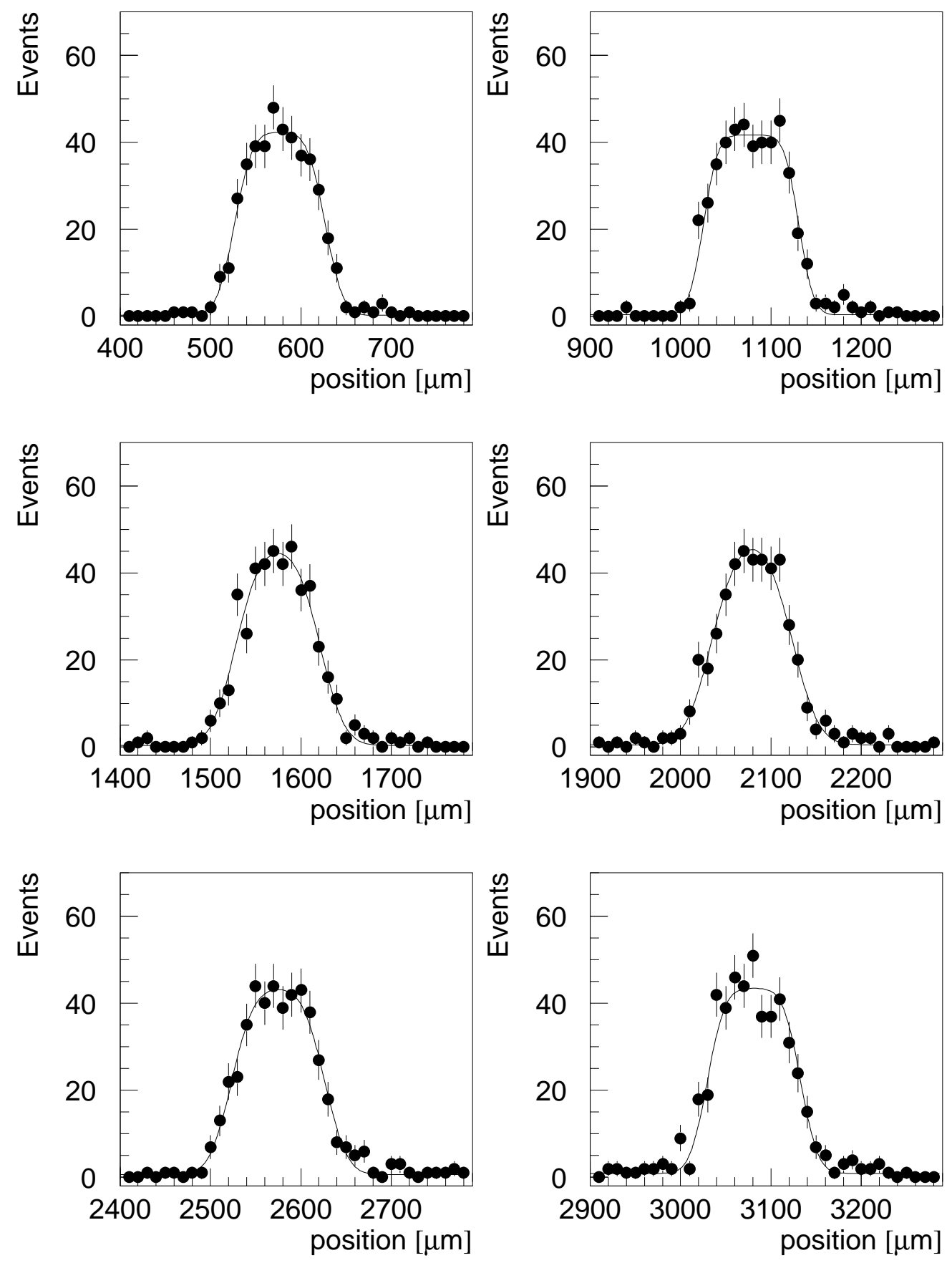

Figure 18: Number of events with a signal greater than noise threshold for a total of 100 events for one strip as a function of the position of the $X$-stage. The plots are taken for different starting positions of the $\mathrm{X}$-ray relative to the $X$-stage. The curves show results from fits explained in the text. 


\section{Scanning of Si Strip}
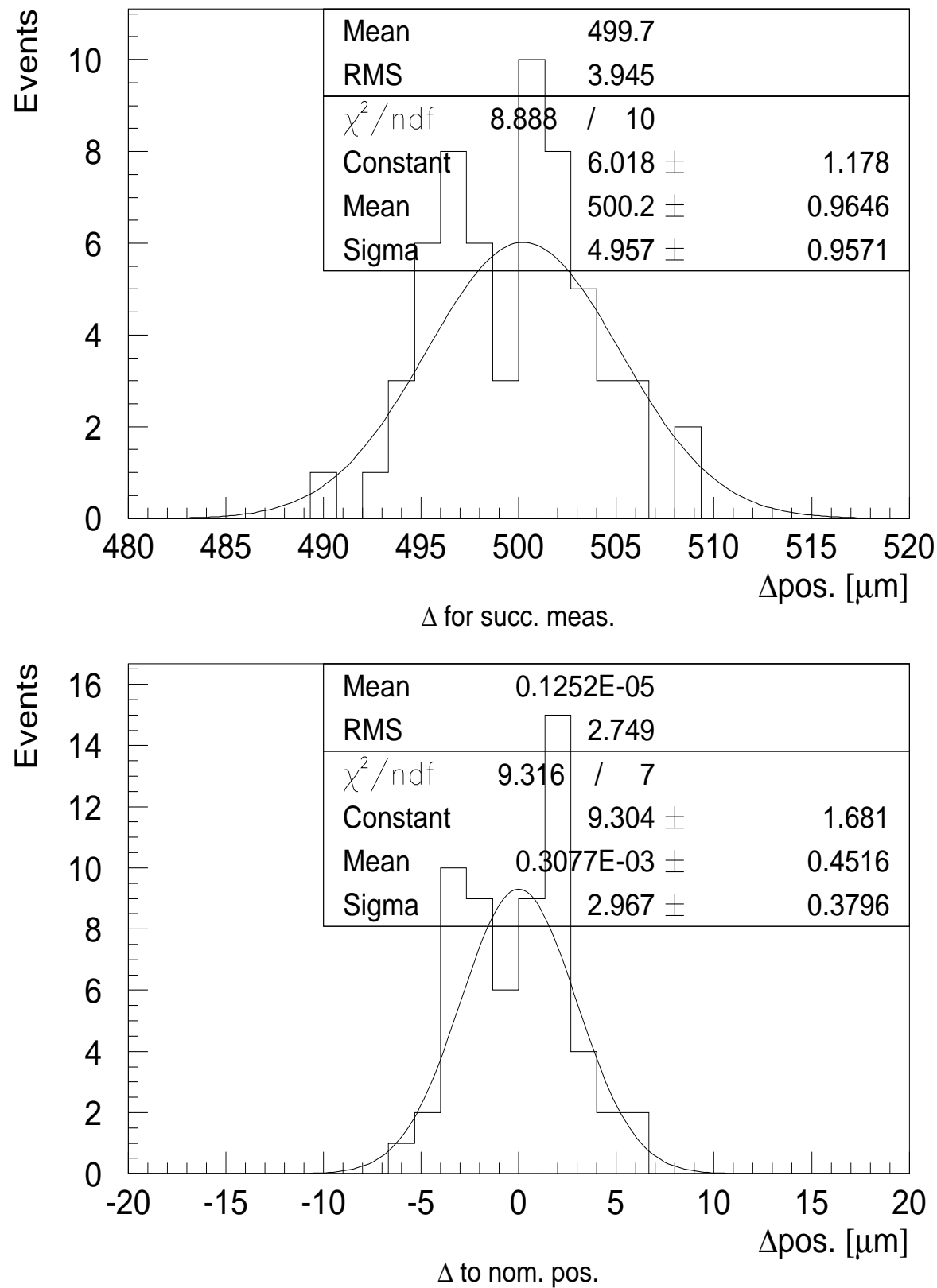

Figure 19: Repeatability of the determination of the strip position using the scanning method. The top plot shows the difference in fitted position between successive scans and the bottom plot the difference to the nominal position. 


\section{Scanning of Si Module}
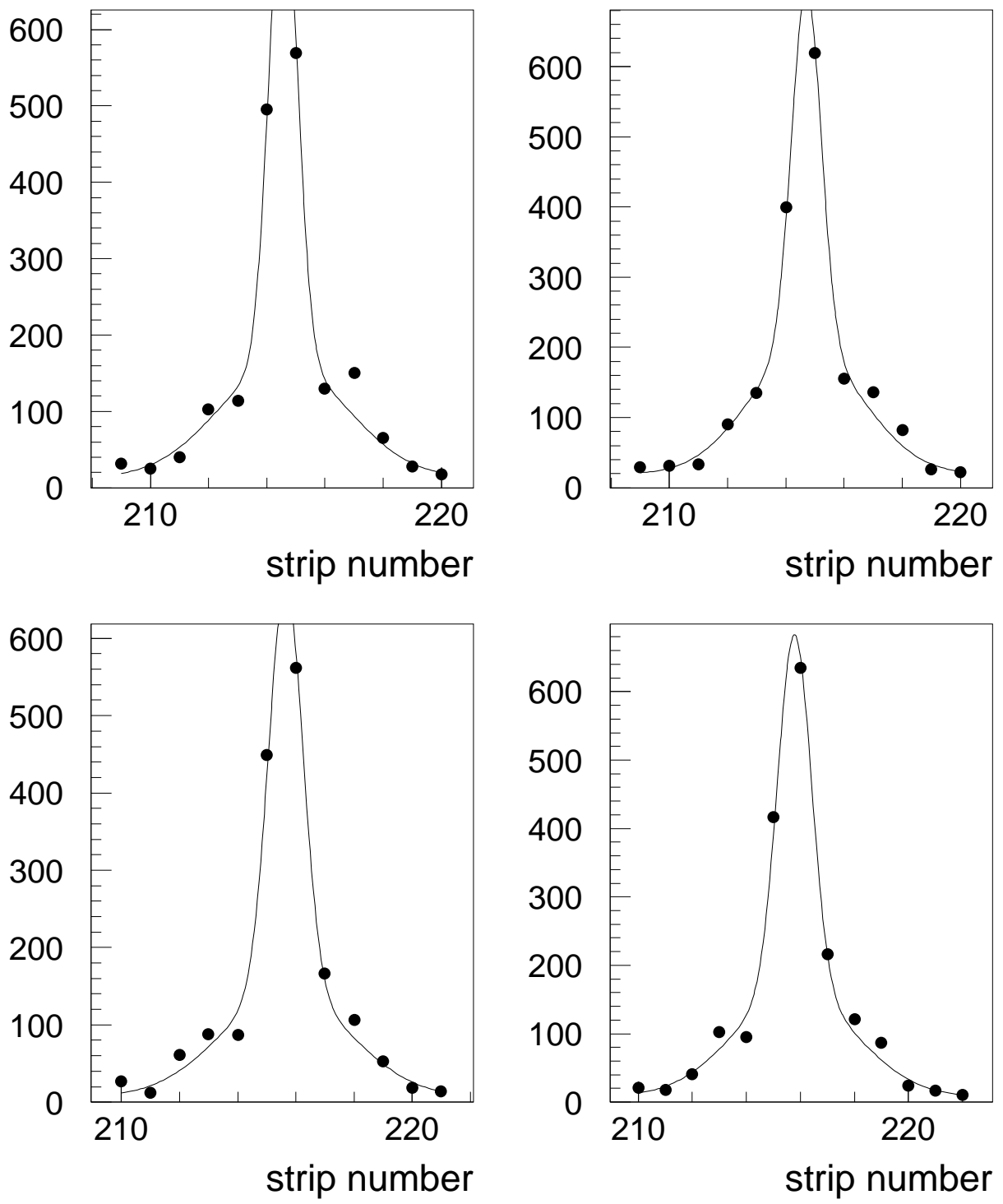

Figure 20: Number of events with a signal greater than noise threshold for a total of 1000 events for fixed position shifted each time by $10 \mu \mathrm{m}$ from the top left to the bottom right plot versus strip channel number. Beam parameters were $V=40 \mathrm{kV}, I=10 \mathrm{~mA}$ and $s=15 \mu \mathrm{m}$. The curve shows the result from a fit with the sum of two Gaussians and a constant. 

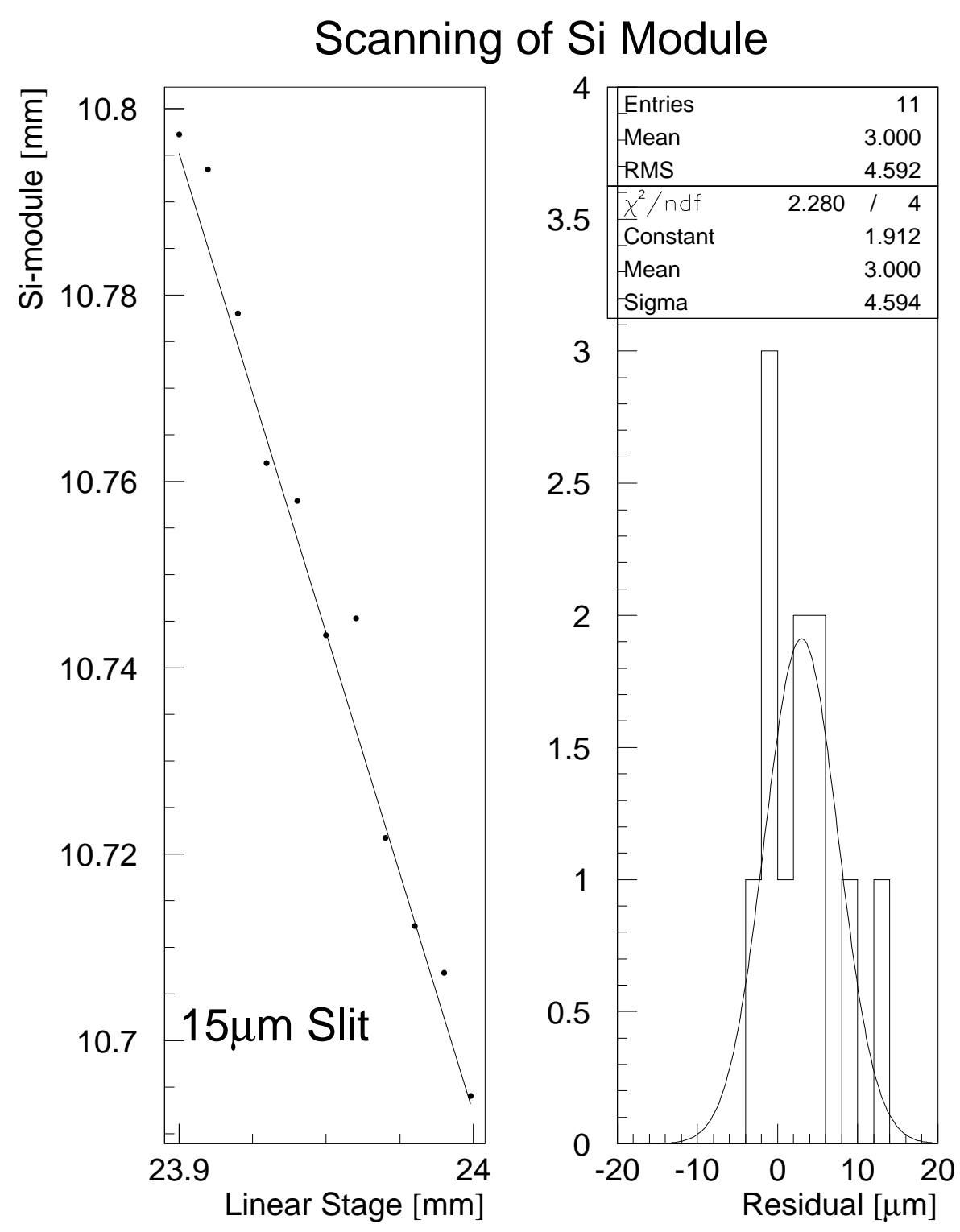

Figure 21: Left: repeating the strip weighting position reconstruction for different positions of the linear stage. The points are fitted with straight line. Right: residual distribution of the shifts from the straight line in the left plot. Measured with a beam with slit width $s=15 \mu \mathrm{m}$. 

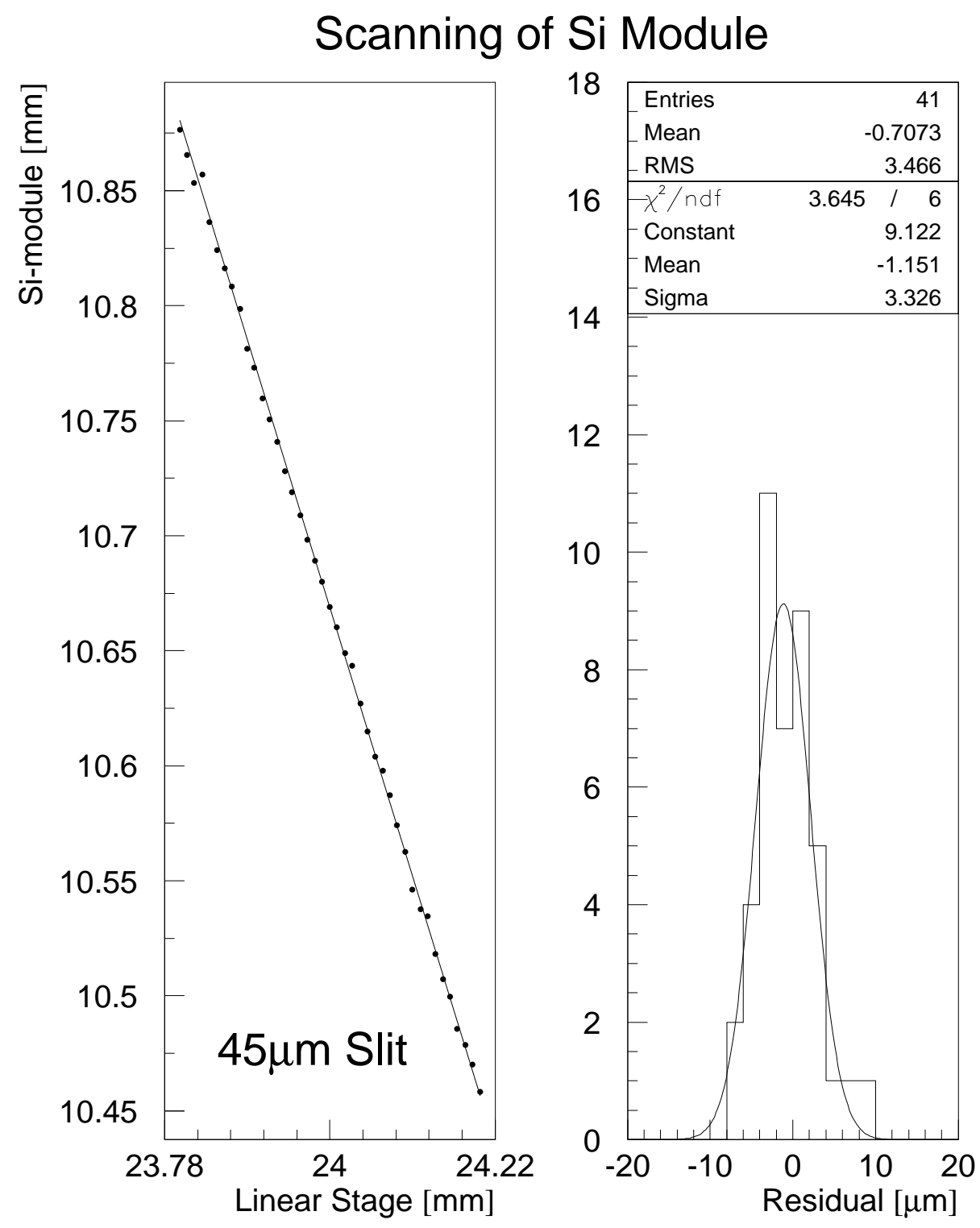

Figure 22: Left: repeating the strip weighting position reconstruction for different positions of the linear stage. The points are fitted with straight line. Right: residual distribution of the shifts from the straight line in the left plot. Measured with a beam with slit width $s=45 \mu \mathrm{m}$. 

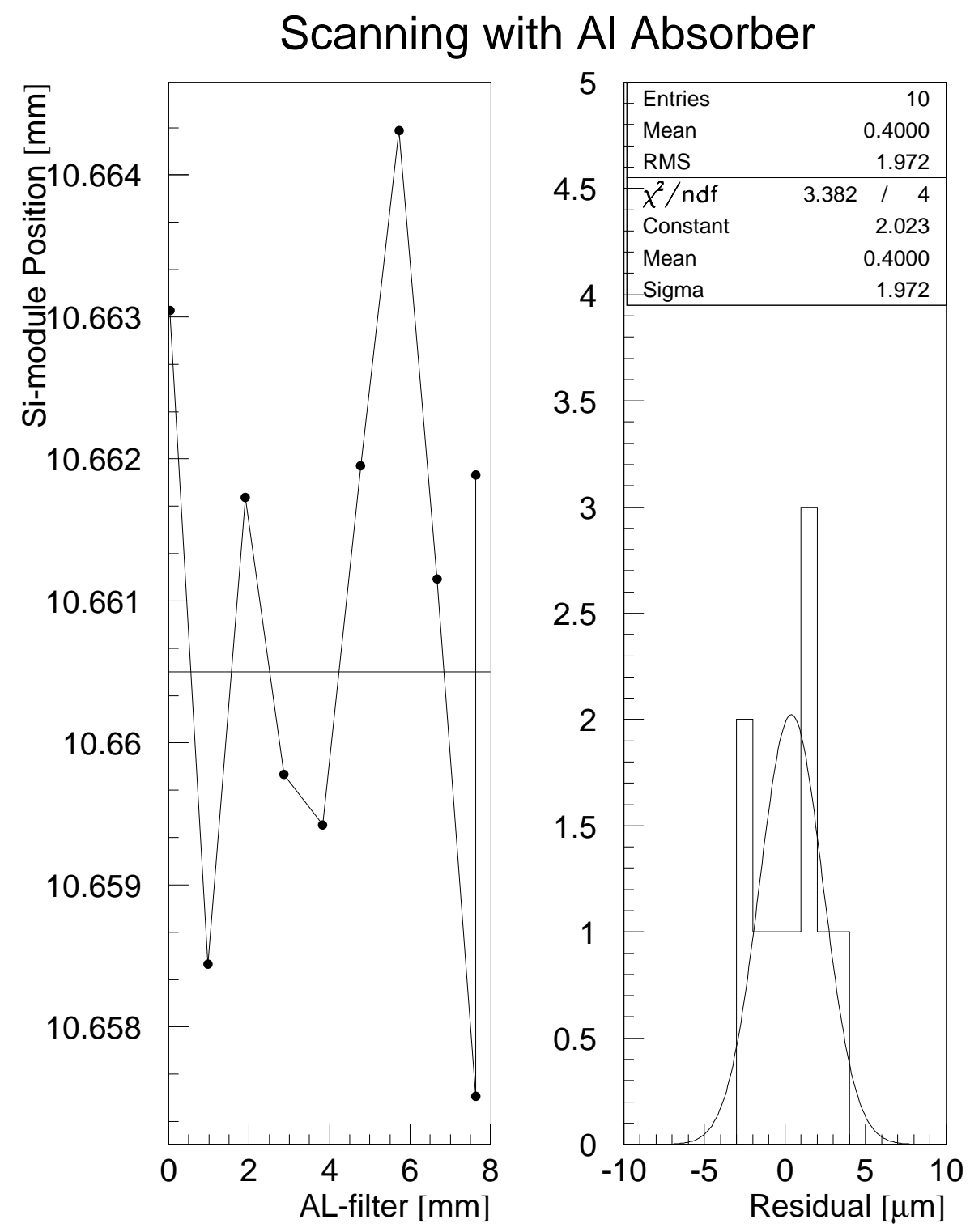

Figure 23: Residual distribution when repeating the strip weighting position reconstruction with increasing thickness of an duraluminium-filter. 

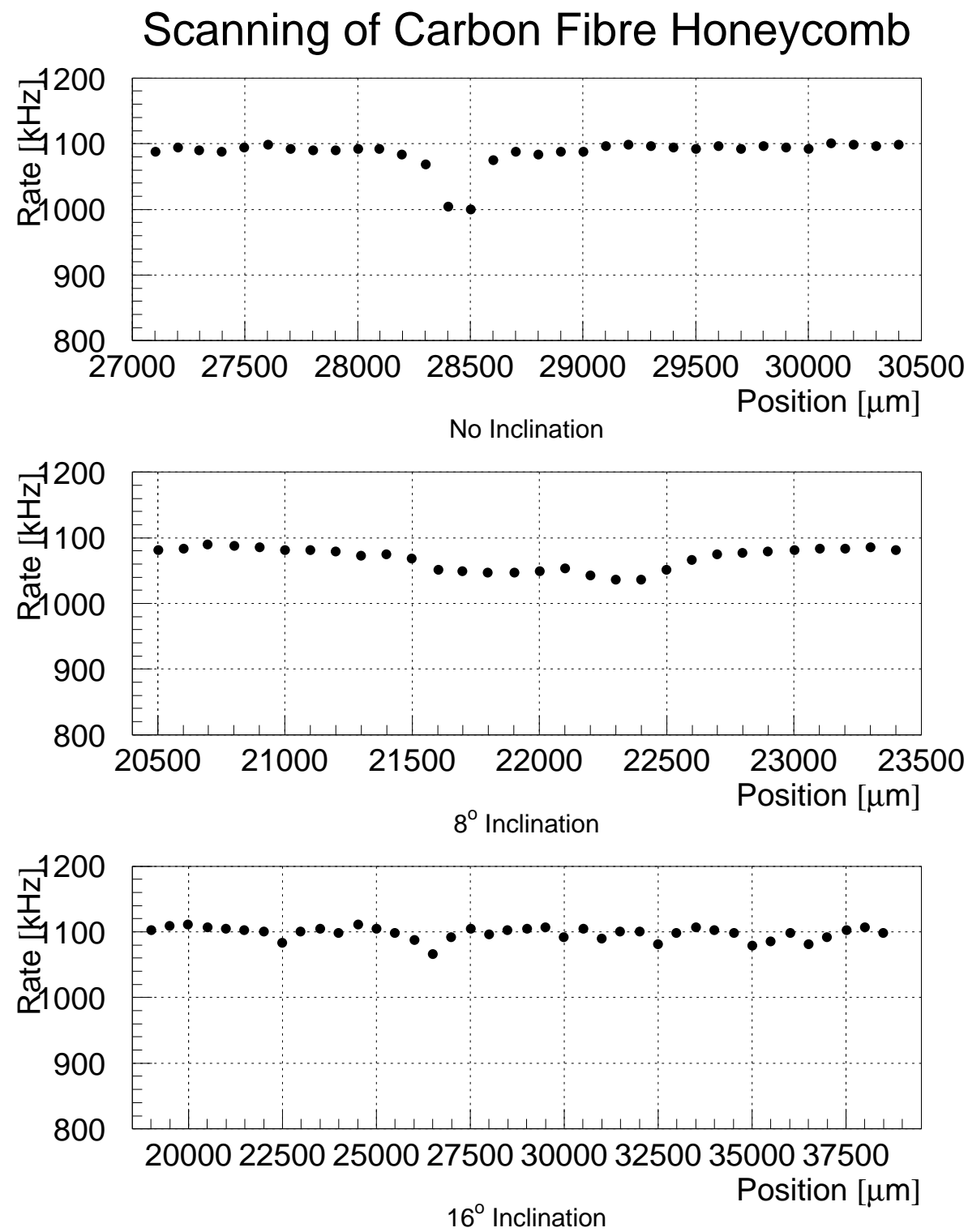

Figure 24: Scanning of a carbon fibre honeycomb structure. Shown is the rate measured in the scintillation counter as a function of the $X$-stage position for different inclination angles of the material. 

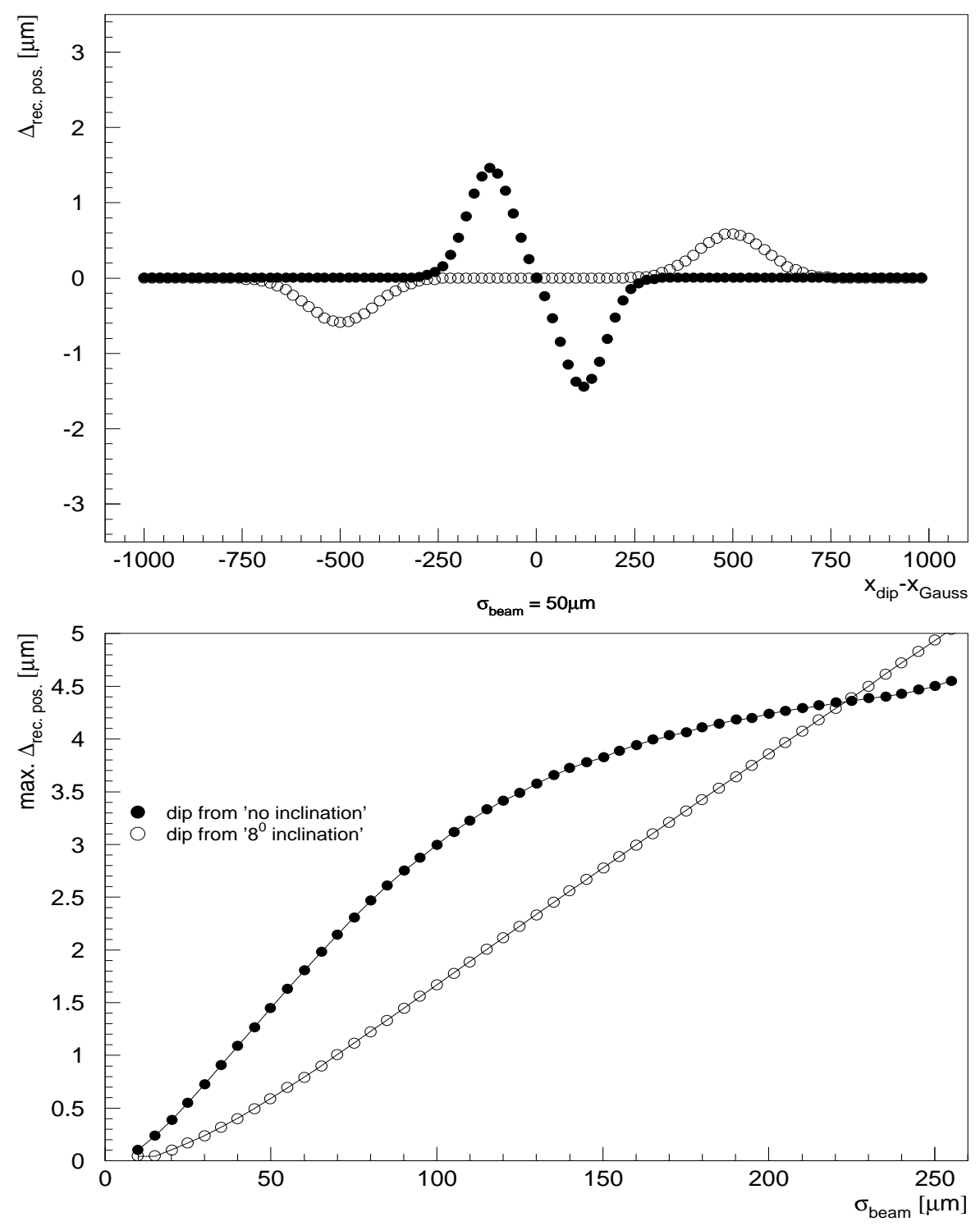

Figure 25: Simulating the effect of absorption in a carbon fibre honeycomb structure on the position reconstruction of Si detectors. The top plot shows the deviation from the nominal position $\Delta_{\text {rec.pos. }}$ expected for a Gaussian beam shape as a function of the relative central position $x_{\text {dip }}-x_{\text {Gauss }}$. The bottom plot shows the maximum deviation as a function of the width $\sigma_{\text {beam }}$ of the Gaussian. Dots show the result for an absorption pattern as measured for the honeycomb with no inclination, circles that for $8^{\circ}$ inclination (see figure 24 ). 
Scanning of Si Strip Inclined by $30^{\circ}$

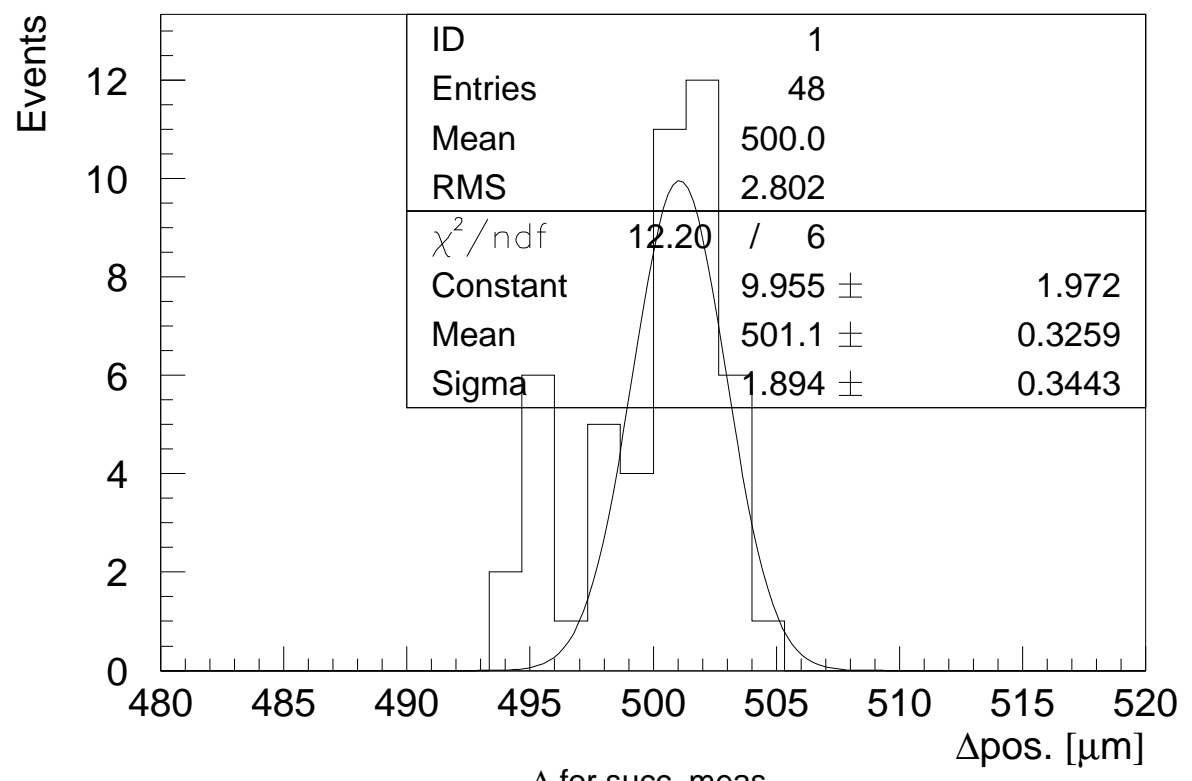

$\Delta$ for succ. meas.

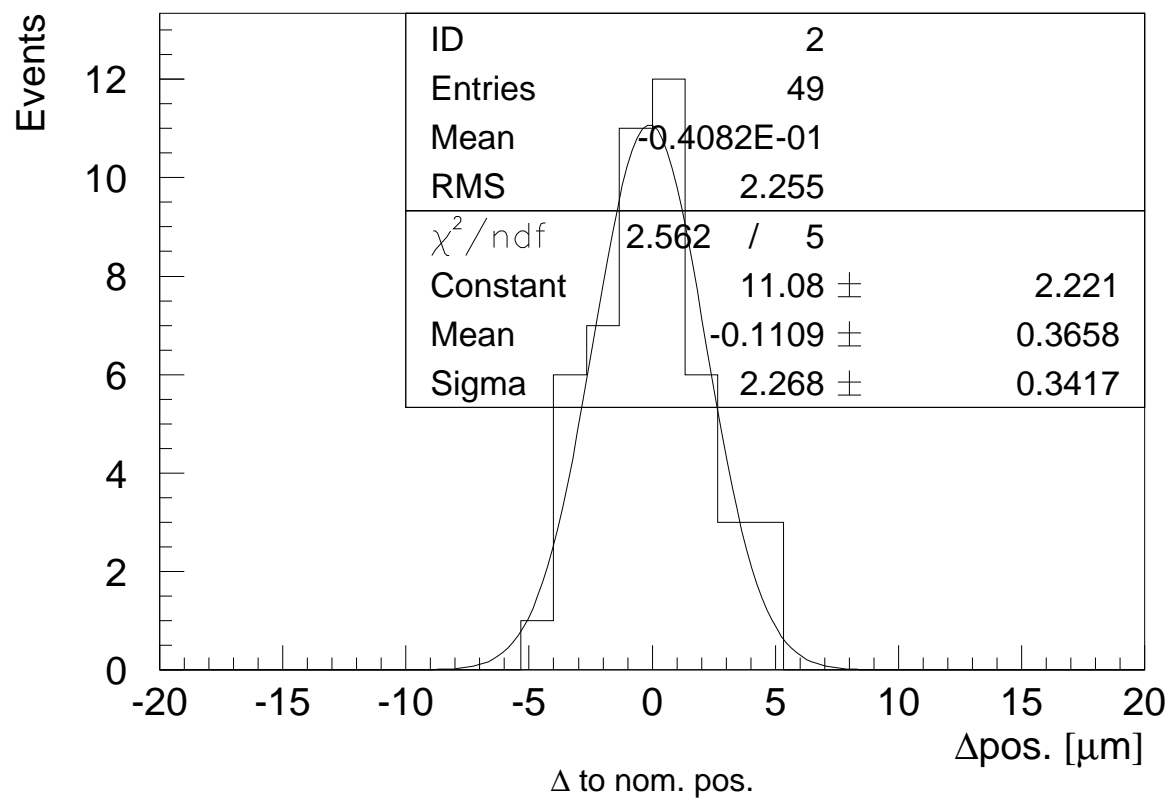

Figure 26: Difference between nominal and reconstructed position for a silicon detector inclined by $30^{\circ}$ showing a repeatability of approximately $3 \mu \mathrm{m}$. 

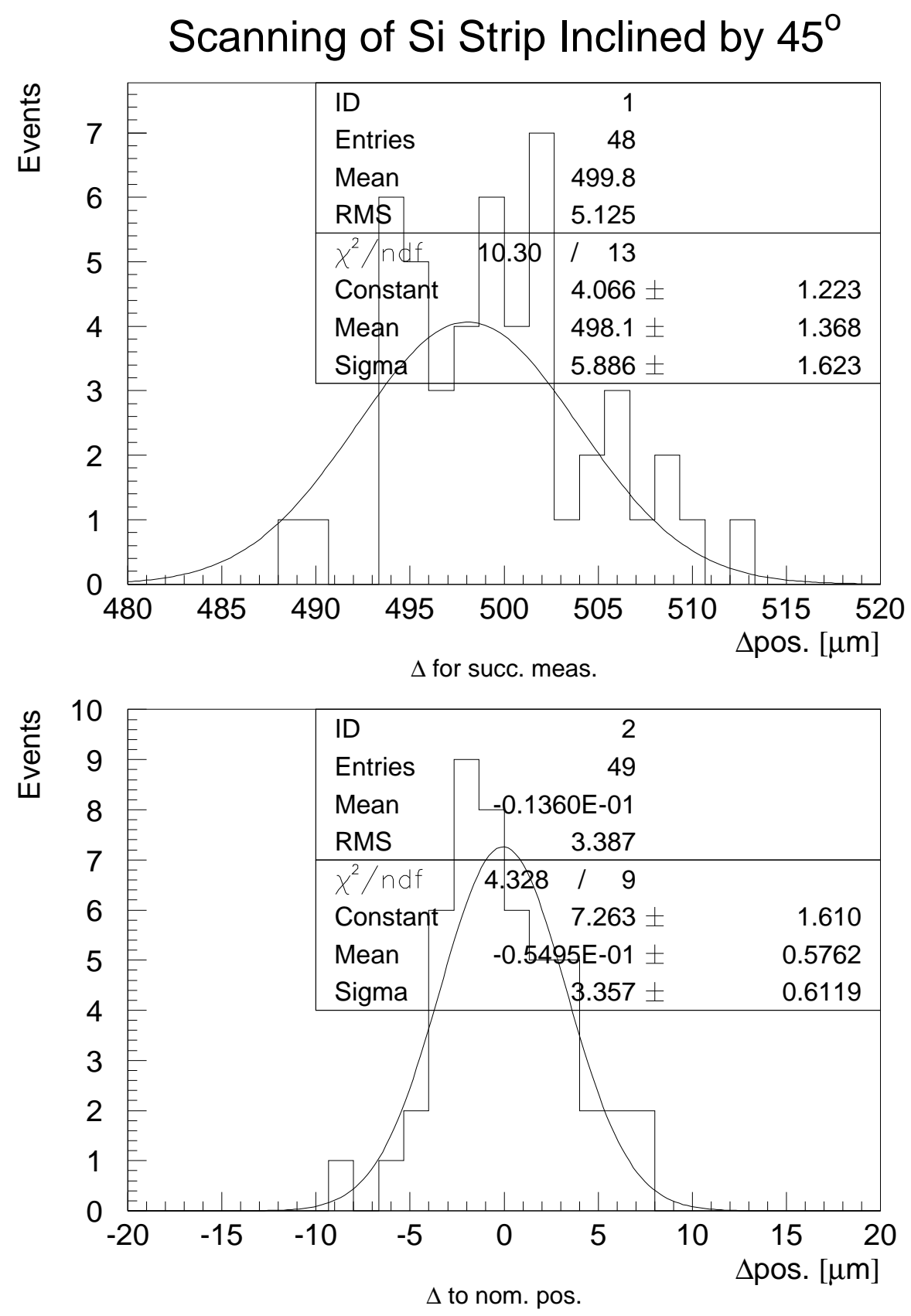

Figure 27: Difference between nominal and reconstructed position for a silicon detector inclined by $45^{\circ}$ showing a repeatability of approximately $5 \mu \mathrm{m}$. 

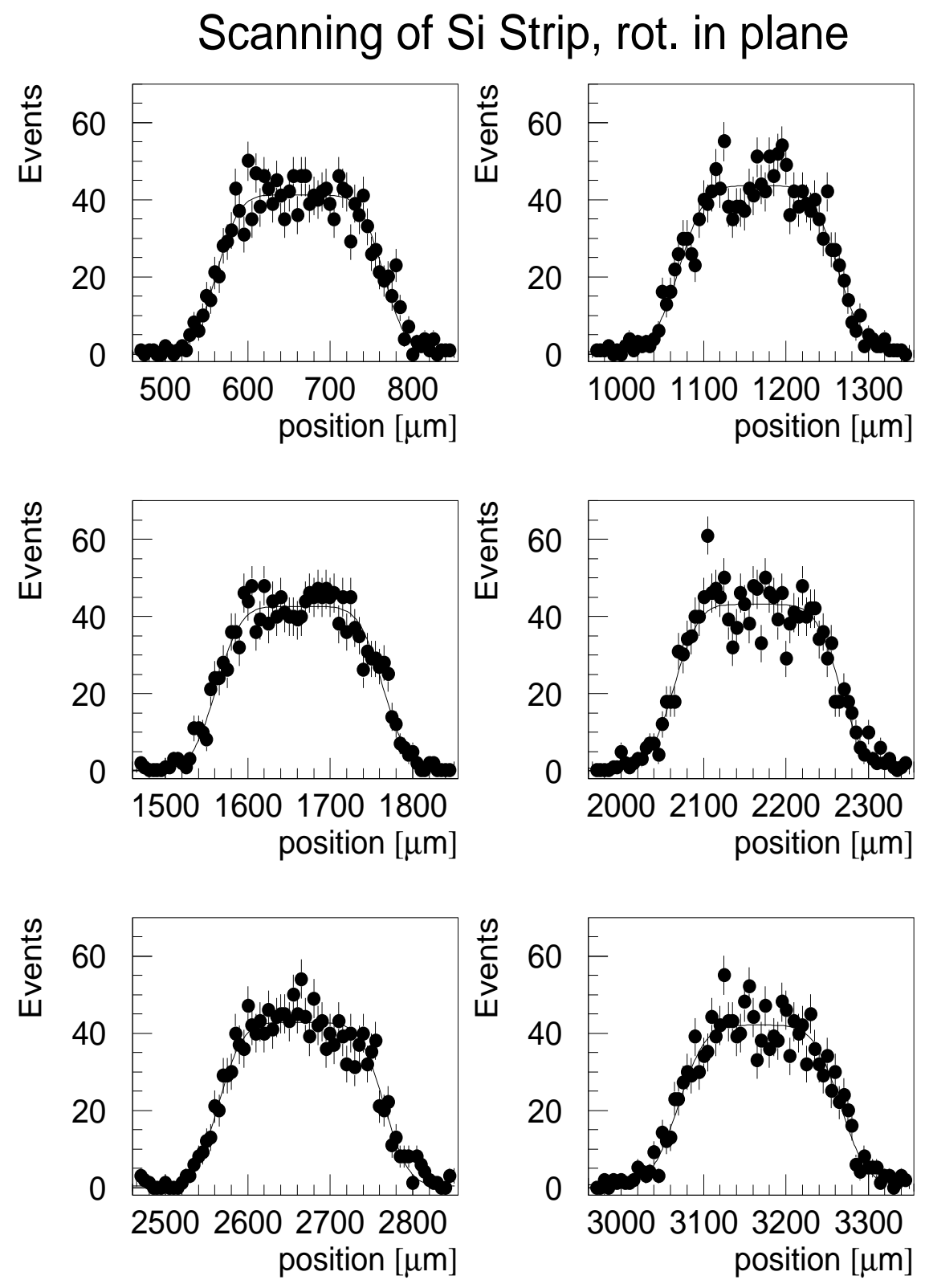

Figure 28: Number of events with a signal greater than noise threshold for a total of 100 events for one strip as a function of the position of the $X$-stage for a silicon detector rotated in the $X Z$-plane by $1.3^{\circ}$. 
Scanning of Si Strip, rot. in plane
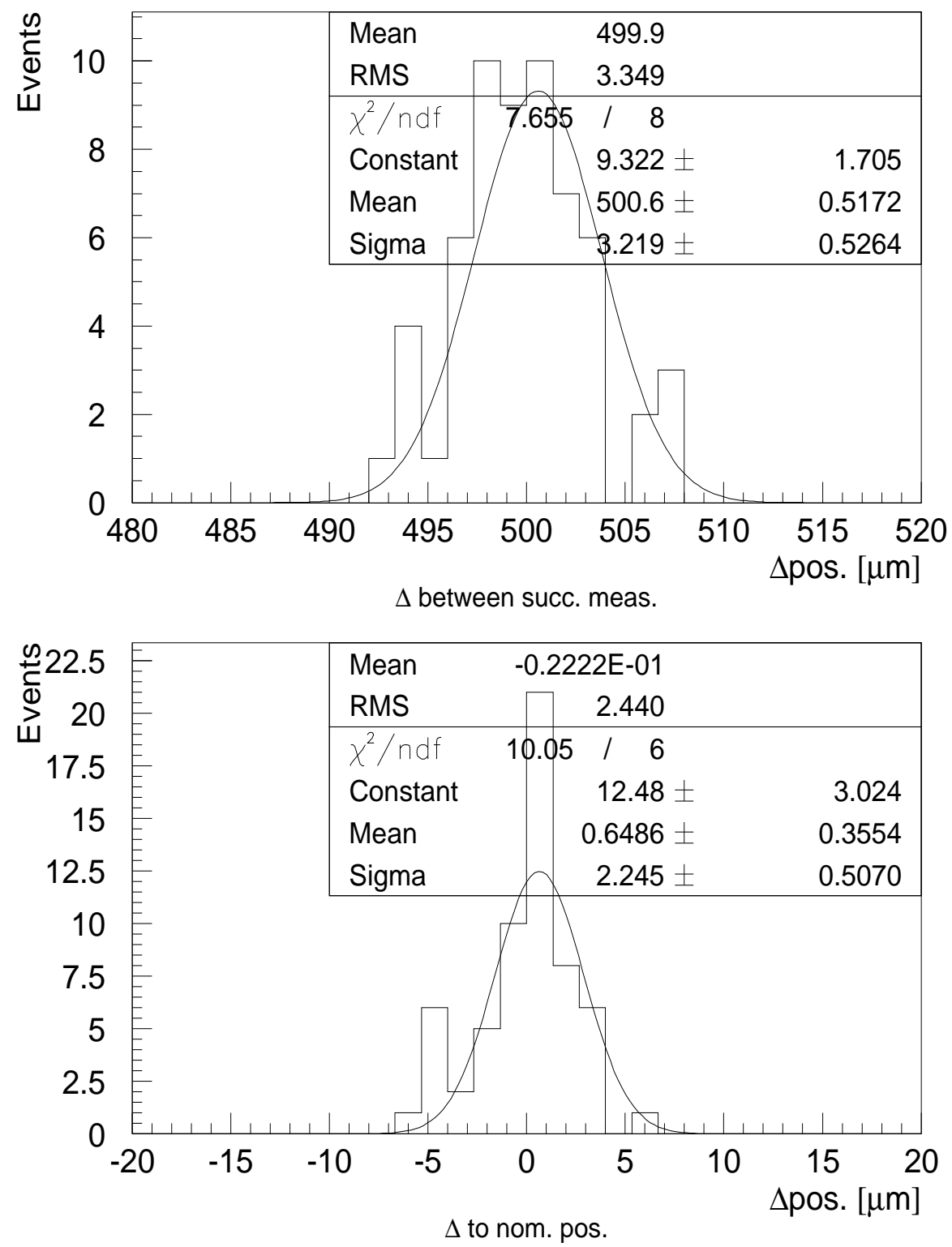

Figure 29: Difference between nominal and reconstructed position for a silicon detector rotated in the $X Z$-plane by $1.3^{\circ}$. 

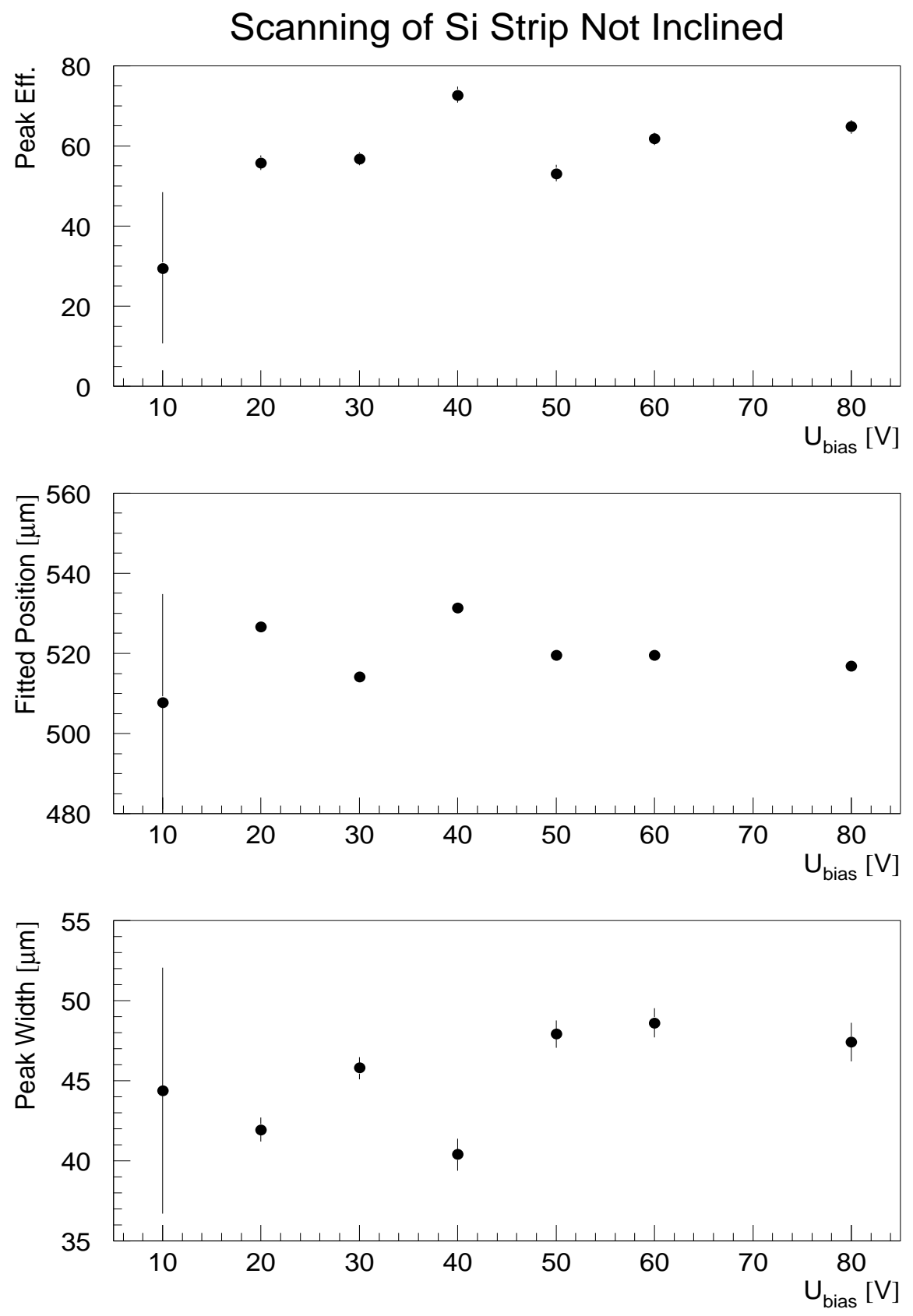

Figure 30: Dependence of the efficiency, reconstructed position and half-width of the rate-peak on the silicon bias voltage $U_{\text {bias }}$. Detector is not inclined. 

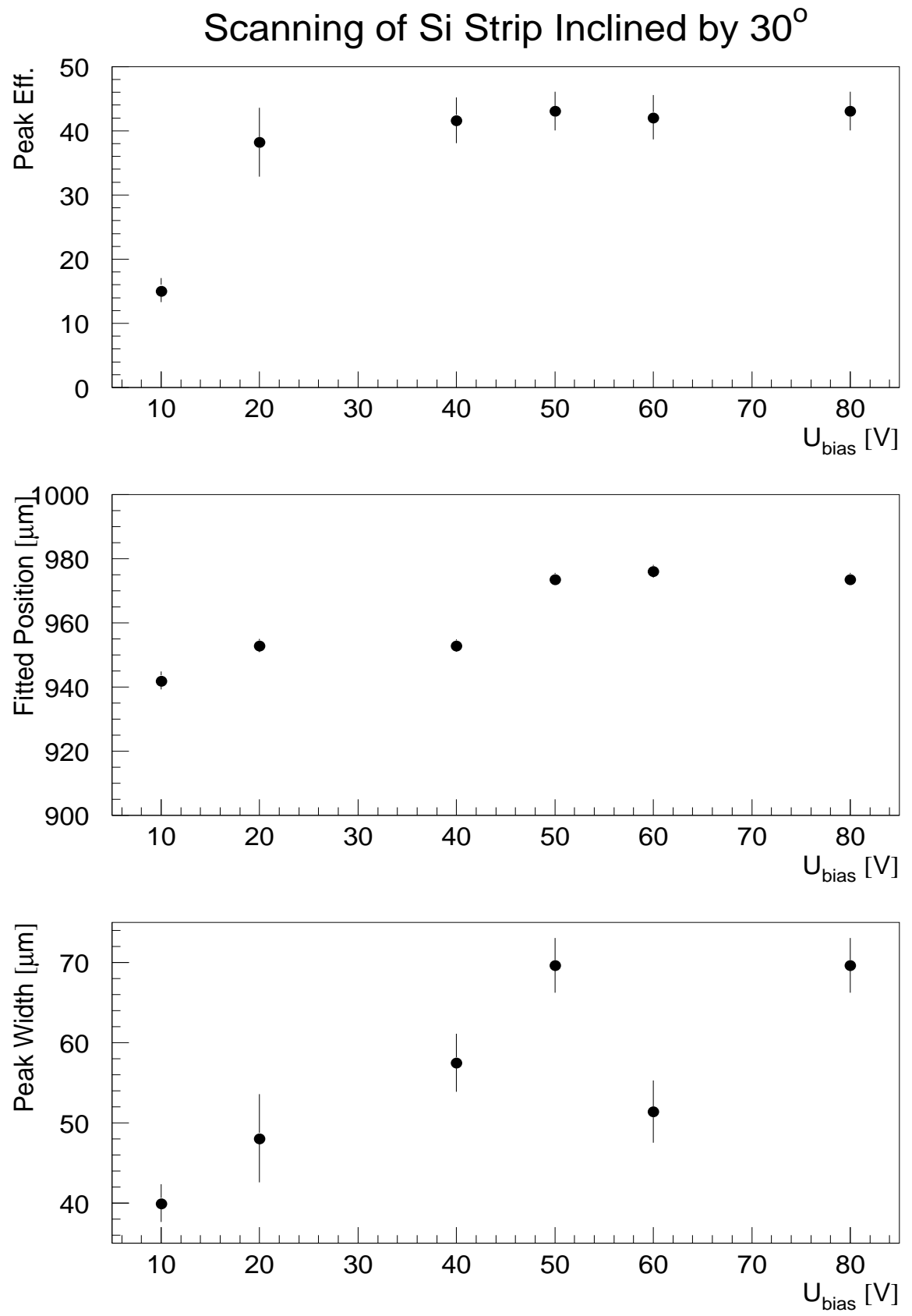

Figure 31: Dependence of the efficiency, reconstructed position and half-width of the rate-peak on the silicon bias voltage $U_{\text {bias }}$ for an inclination of $30^{\circ}$. 
Scanning of Si Strip Inclined by $45^{\circ}$
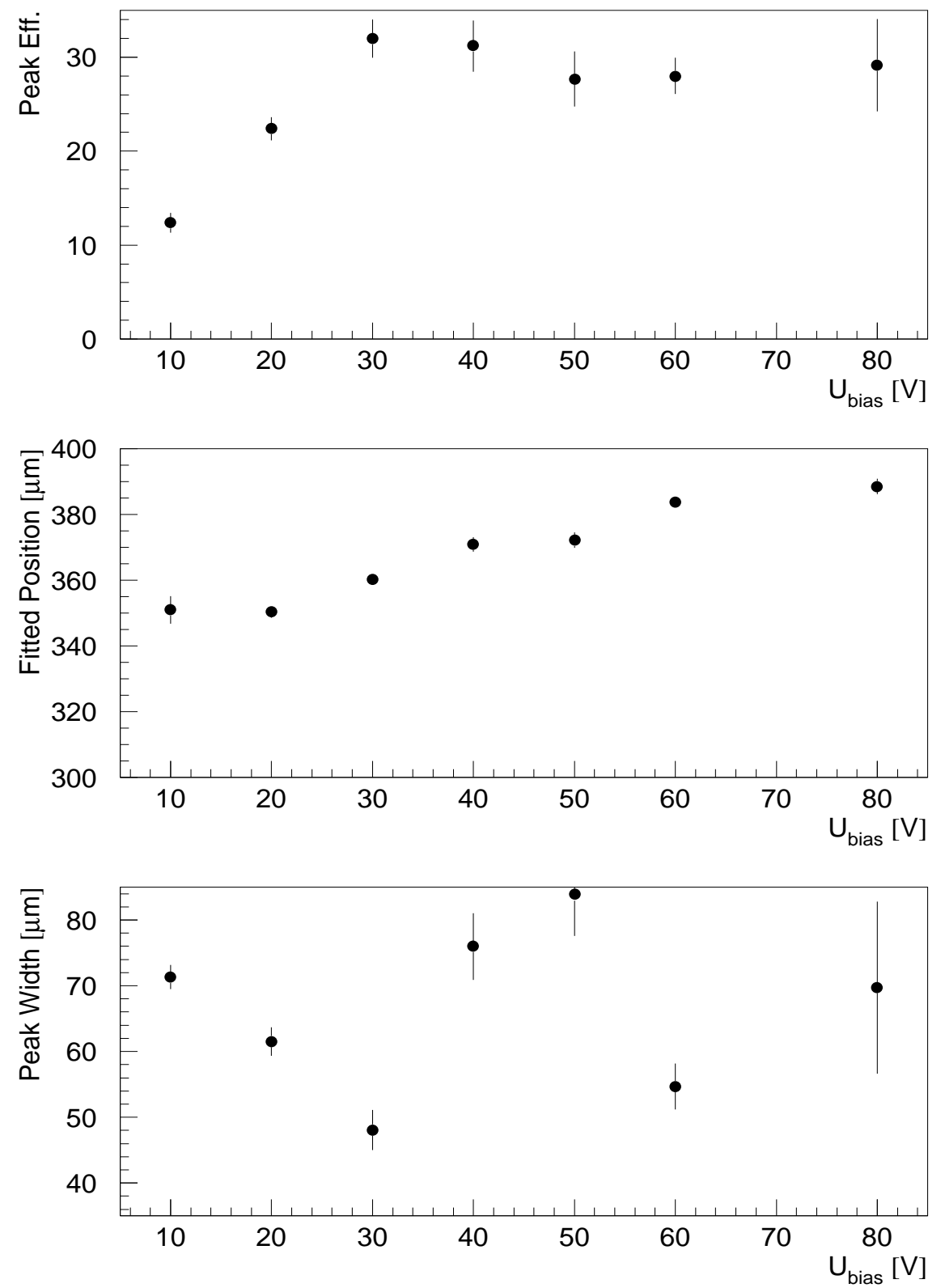

Figure 32: Dependence of the efficiency, reconstructed position and half-width of the rate-peak on the silicon bias voltage $U_{\text {bias }}$ for an inclination of $45^{\circ}$. 

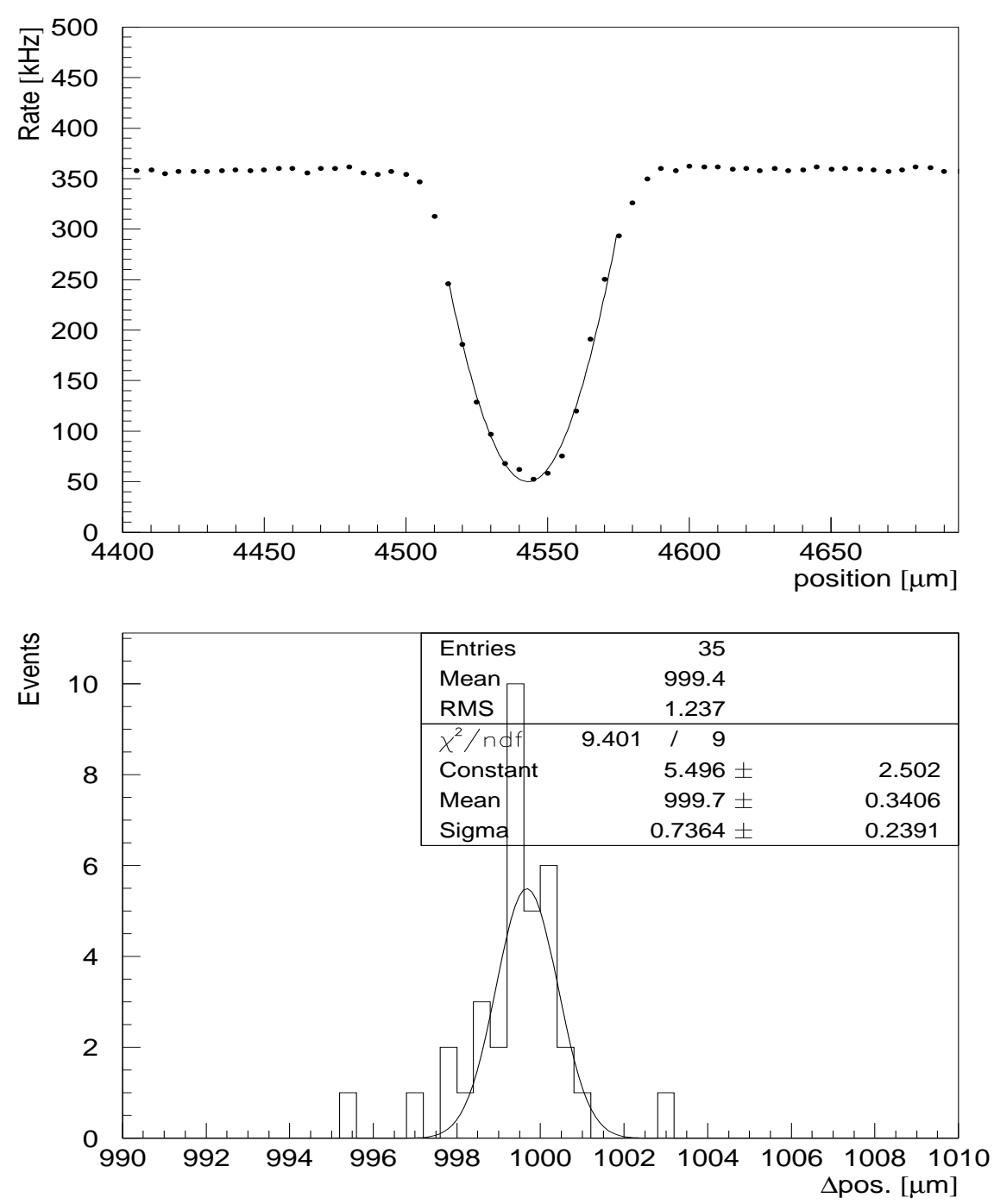

Figure 33: Top: rate measured by the scintillator as a function of the position of a $\mathrm{W}$ wire. The curve is the result of a Gaussian fit. Bottom: difference in the Gaussian peak positions of two successive scans of a W wire with an initial X-ray position shifted by $1000 \mu \mathrm{m}$. 

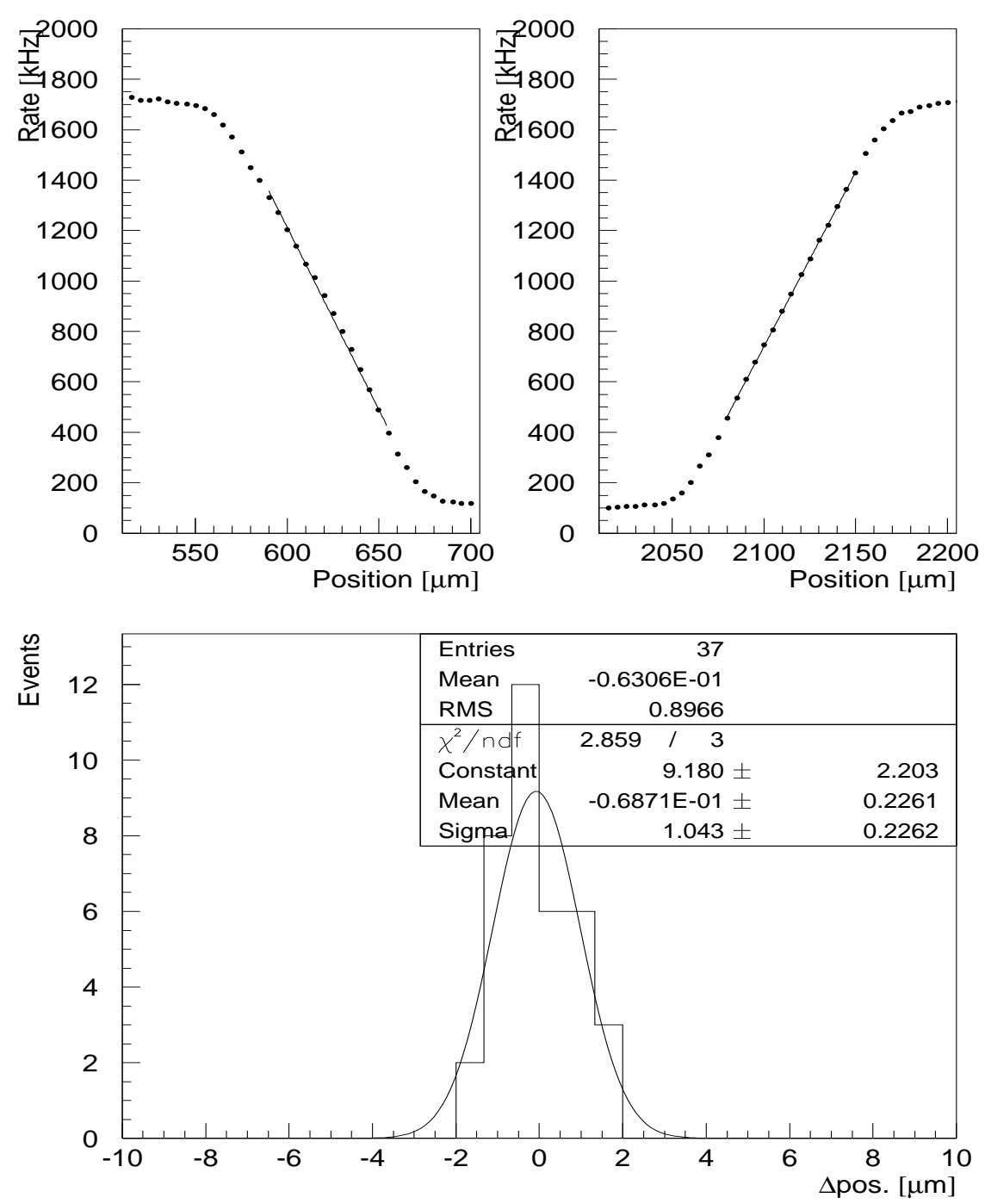

Figure 34: Top: rate measured by the scintillator as a function of the position of a steel pin (left shows the left side and right the right side). The curves are the results of a linear fit to the falling part of the rate. Bottom: difference between measured pin centre and nominal position for several scans. 